\title{
Quality-based and Energy-efficient Data Communication for the Internet of Things Networks
}

\author{
Yasmin Fathy, and Payam Barnaghi, Senior Member, IEEE
}

\begin{abstract}
Large volumes of real-world observation and measurement data are collected from sensory devices in the Internet of Things (IoT) networks. IoT data is often generated in highly distributed and dynamic environments. Continuous transmission of large volumes of data collected between sensor and head/sink nodes induces a high communication cost for individual nodes. This results in a significant increase in the overall energy cost for IoT applications such as environmental monitoring. Decreasing data transmission between nodes can effectively reduce energy consumption and prolong the network lifetime, especially in battery-powered nodes/networks. In this paper, we describe an Adaptive Method for Data Reduction (AM$D R$ ), a data reduction approach for reducing the overall data transmission and communication between sensor nodes in IoT networks such that fine-grained sensor readings can be used to reconstruct the original data within a user-defined accuracy boundary. Evaluation with real-world data shows that AM-DR achieves a communication reduction in some scenarios up to $95 \%$ while retaining a high prediction accuracy. To fully achieve the energy savings enabled by AM-DR, we provide a communication cost model. The proposed model is also integrated into the LEACH protocol to demonstrate how our proposed approach reduces energy consumption and effectively prolongs the network lifetime.
\end{abstract}

Index Terms-Internet of Things (IoT), Sensor networks, Data reduction, Adaptive filters, Data communication

\section{INTRODUCTION}

$\mathbf{T}$ He prevalence of low-cost sensor and actuator devices empowered by their sensing and wireless communication capabilities has created an opportunity for new forms of IoT services and applications. IoT is an umbrella term that refers to the communication and interaction between networkenabled devices (e.g. things) that produce and exchange data about their environment on the network. IoT network is often composed of a large number of sensor nodes being deployed and distributed over a large (dense or sparse) geographical area (i.e. sensing field). This enables capturing data about an observed physical quantity such as temperature, humidity, electric current or light intensity [1].

In typical large-scale distributed and often dynamic and ad-hoc IoT networks for environmental monitoring, resourceconstrained devices send their immediate readings to base stations (i.e. sink nodes) such that base stations respond to user queries within some user-defined accuracy [2]. On the

Y. Fathy is with the Computer Science Department, University College London (UCL) (e-mail: y.fathy@ucl.ac.uk).

P. Barnaghi is with the Centre for Vision, Speech and Signal Processing (CVSSP) at the University of Surrey and the Care Research and Technology Centre at the UK Dementia Research Institute (UK DRI).

Copyright (c) 2019 IEEE. Personal use of this material is permitted. However, permission to use this material for any other purposes must be obtained from the IEEE by sending a request to pubs-permissions@ieee.org other hand, IoT monitoring applications may require the need of (continuous) transmitting large quantities of collected data over long periods of time between the sensor and sink nodes which can be impractical or expensive [3]. Moreover, in detecting a target of interest in a monitoring surveillance area, reducing the number of transmissions between sensors and Fusion Centre (FC) is necessary for improving the network bandwidth efficiency [4]. In such approaches, the FC makes a global decision about the target based on the transmitted local decision on the presence/absence of a target from sensors [5]. Overall, network lifetime and reliability are among the main requirements in different IoT monitoring application scenarios.

The communication and interaction between sensory devices or sensor nodes pose some challenges in IoT networks [1]. Data transmission is a dominant factor of communication overhead and energy consumption in IoT networks. Furthermore, transmitting data between sensor nodes and cluster heads or sink nodes consumes higher energy than data sensing [2, 6].

The communication channel between nodes could also be unreliable. Sensor nodes may fail to transmit their observation data to base stations due to their energy depletion [7]. In several use-cases, it is also impractical to regularly recharge or replace batteries of a large number of deployed sensor nodes. On the other hand, reporting data to sink nodes might also suffer from high latencies in typical large-scale and ad-hoc sensor networks; delays associated with collecting contextual information and energy consumption.

One commonality across IoT monitoring applications is the need to limit the communication between nodes to reduce energy consumption to prolong network lifetime [6]. Prediction-based data reduction techniques have been designed for minimising energy consumption by reducing the amount of data sent by each individual node (i.e. source) to a sink node (e.g. cluster/base node) [8]. These techniques aim at reducing the amount of measurement data sent by each node in the network by exploiting predictive models to predict the measured values both at the source and the sink/base nodes [2, 6]. However, achieving the trade-off between data reduction to reduce transmission between sensor nodes and have adequate quality is a challenging task.

We have developed an Adaptive Method for Data Reduction (AM-DR) [6]. AM-DR is based on a convex combination of two decoupled Least-Mean-Square (LMS) windowed filters with differing sizes. The filters aim at estimating the next sensor readings both at the source and the sink nodes such that sensor nodes require transmitting only their readings if those deviate significantly (with a pre-defined quality) from the prediction. 
In this paper, we have extended and improved our work proposed in [6] by providing a communication cost model, mediating between predicting quality and energy efficiency and allowing end-users to maximise network lifetime based on their defined data quality. Moreover, we extend the AM-DR such that Cluster Heads (CHs) opt dead/failed nodes out from their prediction models. We have also conducted experiments with real-world datasets to demonstrate the applicability and scalability of our proposed solutions. To fully achieve the energy savings enabled by AM-DR in terms how many sensor nodes can be alive, stability period (i.e. the time interval before the first node is dead) and other criteria, we also integrate our approach into LEACH (Low-Energy Adaptive Clustering Hierarchy) protocol [9].

The paper is structured as follows. The problem formulation is explained in Section III. Section III provides the required background and related work. Our proposed algorithm and its energy model are discussed in Section IV] The performance evaluation including datasets, parameter settings, reproducibility of experimental results and evaluation criteria descriptions are included in Section V In Section VI the proposed algorithm is evaluated and analysed on real-world datasets against the state-of-the-art. We also integrate our approach into the LEACH protocol to demonstrate how our proposed solution reduces energy consumption in IoT sensor networks. We conclude the paper and explain the future directions of our research in Section VII.

We have summarised our most common parameters that are used for the equations as follows. $x(t)$ is the actual sensor value and $\hat{y}(t)$ is the predicted sensor value (i.e. the output of the filter) at a time step $t$. We denote $e(t)$ as the error between the actual and estimated data values $(\hat{y}(t)-x(t))$. The output of the filter $\hat{y}(t)$ depends on applying the filter weight $w(t)$ on the actual data value. The filter weight (i.e. coefficient) is adapted with a learning rate $\alpha$, for minimising the error $e(t)$. Moreover, the filter weight $w(t)$ is the combination of two filter weights, based on $\lambda(t)$ which is is a mixing scalar parameter such that $0 \leqslant \lambda(t) \leqslant 1$ to preserve the convexity of the combination between filters' weights.

\section{PROBLEM FORMULATION}

Given an IoT based wireless sensor network of $N$ sensor nodes that are randomly deployed in a monitoring region (i.e. sensing field). Sensor nodes are the source of information such that at each time interval $t>0(t=1,2, \cdots, T)$, each sensor node $S_{i}(i=1,2, \cdots, N)$ collects data streams $x_{i, t}$ ( $i$ is the sensor node index and $t$ is the time instance) about observed phenomena (e.g. physical quantities) such as temperature. Sensor nodes (which we call non-CHs) transmit data to Cluster Heads (CHs). $\mathrm{CH}$ nodes collect, aggregate and forward data received from non-CH nodes to a Base Station (BS) (i.e. a gateway) for further processing. Fig. 1 gives an illustrative example of a network architecture where there are two $\mathrm{CHs}$ and one BS. In this example, each $\mathrm{CH}$ has a set of 6 connected nodes. $\mathrm{CH}$ aggregates data transmitted by its connected sensors and forwards it to the BS.

We are interested in addressing WSN requirements by reducing energy consumption and prolonging system lifetime such that communication cost for each individual node is reduced. Taking the characteristics of WSN into consideration, we assess the problem by tackling the following questions:

1) Data reduction: How much data can be sent from sensor nodes to $\mathrm{CH}$ or $\mathrm{BS}$ while achieving a considerable reduction in data transmission?.

2) Data quality: How much fine-grained sensor readings should a source (e.g. sensor node) send such that original readings can be reconstructed within a user-defined quality (i.e. maximum absolute deviation/minimum accuracy) at a destination (e.g. CH, a base station)?

3) Energy savings: How much energy depletion can be reduced in the network?

Addressing these questions effectively allows reducing the number of data transmissions between sensor and sink or $\mathrm{CH}$ nodes by avoiding unnecessary or redundant transmissions without affecting the quality of sensor measurements. Overall, we aim at improving upon our work proposed in [6] by the following:

- Analysing AM-DR energy model where energy requirement of each individual sensor node is quantified. We have modified first order radio model [9, 10] to demonstrate radio characteristics in terms of energy dissipation of transmitting, receiving and processing modes for each sensor node.

- Integrating our approach into a common routing protocol to demonstrate how AM-DR can effectively prolong network lifetime.

- Extending AM-DR to address the node failure problem such that $\mathrm{CH}$ is able to determine dead/fault sensor nodes.

- Demonstrating AM-DR capabilities for enabling applications to achieve a trade-off between data quality for prediction and communication overhead of each individual sensor node by sending granular data that enables original observation data to be reconstructed within some user-defined quality.

- Conducting a set of experiments on real-world datasets including a dataset with drifts to show how AM-DR adapts well with dynamic changes in sensor measurements and is compatible with applications requiring strict guarantees on data quality.

\section{BACKGROUND AND RELATED WORK}

This section briefly discusses some of the existing work in this domain and describes the background information.

\section{A. LMS algorithm and adaptive filters}

Least-Mean-Square (LMS) is a de facto adaptive filtering algorithm that has a low computational overhead [2]. LMS is mainly based on estimating a set of filter weights (i.e. coefficient) by utilising a stochastic gradient descent approach in which weights are updated with an aim to minimise the mean square error $e(t)$ of the filter at each time instance $t$. The mean square error is defined as the difference between the actual and the estimated (i.e. filter output) data values.

$$
e(t)=x(t)-\hat{y}(t)
$$




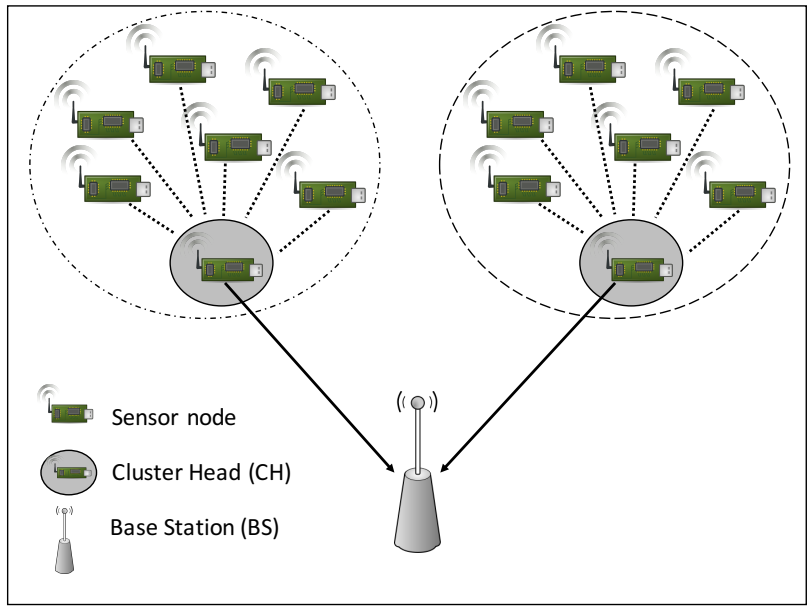

Fig. 1. Example of an IoT based WSN

where $x(t)$ is an observation data at a time $t$ and $\hat{y}(t)$ is the output of applying an adaptive filter to the input $x(t)$ such that

$$
\hat{y}(t)=w(t) x(t)
$$

$w(t)$ is the filter coefficient that is adapted to minimise the error $e(t)$ with a learning rate $\alpha$ (i.e. step size ) using standard LMS rule.

$$
w(t)=w(t-1)+\alpha e(t) x(t)
$$

Some existing works have utilised a combination scheme of two adaptive filters such as [11, 12, 13, 14] where a convex combination of two independent and simultaneous adaptive filters are applied on same data input (e.g. $x(t))$ to improve the steady-state and performance of LMS. In such a case, the weights of the two filters are combined using a parameter $\lambda(t) . \lambda(t)$ is a mixing scalar parameter such that $0 \leqslant \lambda(t) \leqslant 1$ to preserve the convexity of the combination between filters' weights [14]. The overall weight of $w(t)$ as a mixture of two filter weights can be represented as follows:

$$
w(t)=\lambda(t) w_{1}(t)+(1-\lambda(t)) w_{2}(t)
$$

where $w_{1}(t)$ and $w_{2}(t)$ are the weights of the first filter and the second filter at a time instant $t$, respectively. The convex combination parameter $\lambda(t)$ is updated with a step size of $\alpha$ using the standard LMS adaptation rule [15, 16] similar to equ. 3 as follows:

$$
\lambda(t+1)=\lambda(t)+\alpha e(t) x(t)
$$

Similar to the same idea of the convex combination of two filters, a change detection approach based on a combination of two models have been proposed in [17]. The approach relies on Long-Term (LT) memory and Short-Term (ST) memory models to detect change-points based on using a growing window for LT model and a fixed window size for ST model in order to obtain a better change detection using the collaboration between the two models.

\section{B. Dual prediction}

Sensory devices consume a huge amount of energy for communicating and exchanging data with each other. Optimising the communication channel between sensors is a key factor for reducing energy consumption in IoT networks [18]. To this end, IoT networks shifts towards developing local approaches for reducing data transmission and avoiding latency issues.

Several works applied data reduction strategies on data collected by sensor nodes for reducing data transmission in sensor networks such as [19], [2] and [20]. Data reduction is often achieved by utilising predictive models that aim at predicting the current measured values of sensor nodes from their already delivered data to a BS or a $\mathrm{CH}$. In this case, readings that can be predicted (according to some criteria such as accuracy) do not need to be reported, and this results in reducing data transmission between nodes and a central server.

Most of the existing work is based on adaptive filtering techniques for sensor data prediction [18, 21]. This paradigm is known as "dual prediction scheme" [2]. Dual prediction scheme is a potential candidate for optimising data transmission in sensor networks [8]. In dual prediction scheme, prediction models are constructed at both the sensor and the sink nodes such that sink nodes use the historical readings they received from sensor nodes for expecting their coming readings. Based on the difference between the prediction and the actual readings, sensor nodes decide whether or not they should transmit their observations to a central server (e.g. a gateway or a base station). Similarly, the same situation can be considered between sensor nodes and $\mathrm{CH}$; in principle, it depends on the network architecture or topology. Interested readers can refer to Dias et al. [22] to study the impact of data prediction schemes on the reduction of the number of transmissions in WSN.

Jain et al. [23] propose a dual prediction scheme that exploits Kalman filters for predicting coming sensor readings. However, Kalman filters rely on pre-existing knowledge about sensor data models (e.g. statistical data properties or data distribution) as a priori. To address this issue, several works have considered using LMS adaptive algorithm in various dual prediction schemes such as [2], [24], [25] and [26]. For instance, Santini and Romer [2] propose a dual prediction approach based on LMS filters that requires no prior knowledge. Their approach achieves up to $92 \%$ communication reduction such that the predicted sensor readings have a deviation of \pm 0.5 from actual readings on a real-world (office environment) temperature dataset. However, the existing models are based on single LMS filters that have limitations on adaptability to capture both slow and fast changes in sensor data [21].

Some clustering-based protocols mainly focus on extending the network lifetime from the perspective of $\mathrm{CH}$ selection and cluster formation such as LEACH. LEACH [9, 10] is a well known adaptive cluster-based protocol for sensor networks. LEACH assumes that all sensor nodes are homogeneous in terms of their energy. Sensor nodes are organised into cluster head nodes (i.e. CHs) and cluster members (i.e. sensor nodes) such that cluster members transmit their sensor readings to $\mathrm{CH}$ that incorporates data aggregation for reducing the transmis- 
sion to $\mathrm{BS}$. In such a case, $\mathrm{CH}$ nodes exhaust its battery and die quickly more than normal sensor nodes. LEACH uses a randomised rotation of $\mathrm{CHs}$ such that a sensor node becomes $\mathrm{CH}$ based on a probability $p$ that depends on the number of clusters and whether or not the sensor node has already been a $\mathrm{CH}$ within $\frac{1}{p}$ rounds.

LEACH-based clustering protocols mainly prolong the network lifetime from the perspective of $\mathrm{CH}$ selection and cluster formation [27]. To optimise communication between sensors in IoT networks, it is also essential to reduce data transmission and generation in the network. We integrate our AM-DR and its energy model into LEACH to demonstrate how AM-DR can effectively prolong network lifetime from the perspective of data transmission; however, our model can be incorporated into other cluster-based protocols.

\section{OUR APPROACH}

This section briefly demonstrates our initial AM-DR strategy [6] and its extension to address the node failure problem such that $\mathrm{CH}$ is able to determine dead/fault sensor nodes. It then analyses AM-DR energy model where energy requirement of each individual sensor node is quantified.

\section{A. Quality-based and energy-efficient approach}

Our Adaptive Method for Data Reduction (AM-DR) relies on a dual prediction scheme for reducing the number of data transmissions in WSN. AM-DR exploits a convex combination of two LMS adaptive filters (Algorithm 1). We have shown in our previous work in [6] that AM-DR has high predictability for upcoming sensor reading. It also adapts well to the changes in temperature sensor measurements compared to the work presented by Santini and Romer [2].

In AM-DR, data prediction model relies on a construction of an initial identical predictive model at both the $\mathrm{CH}$ and sensor nodes to describe data evolution. It is worth noting that each $\mathrm{CH}$ or BS (according to the network structure as mentioned earlier) should run AM-DR for each of its connected nodes. Fig. 2 shows AM-DR model. The model predicts the coming sensor readings using LMS filters (i.e. $\hat{y}(t)$ ) for each sensor node $S_{i}(i=1,2, \cdots, N)$ with a certain defined quality bounds (i.e. minimal accuracy $\left|e_{\max }\right|$ ) comparing to other existing approaches including [2]. If the quality is satisfied considering the given boundary, the query issued by a user is evaluated and answered using the constructed predictive models at $\mathrm{CH}$ without the need to communicate with the sensor nodes. Furthermore, if the model is not accurate enough (e.g. underlying sensor readings have been changed significantly), direct communication is required between sensor nodes and $\mathrm{CH}$ for updating the models at $\mathrm{CH}$. In such a case, the actual readings (i.e. $x(t)$ ) from sensors have to be retrieved, and the predictive model has to be updated at both the $\mathrm{CH}$ and sensor nodes. It is worth mentioning that the same situation can also be applied to the communication between $\mathrm{CH}$ and $\mathrm{BS}$.

Our approach does not only require any prior knowledge, but also achieves a better communication reduction while retaining a high quality for predicting next sensor readings using LMS windowed filters with differing sizes. As mentioned

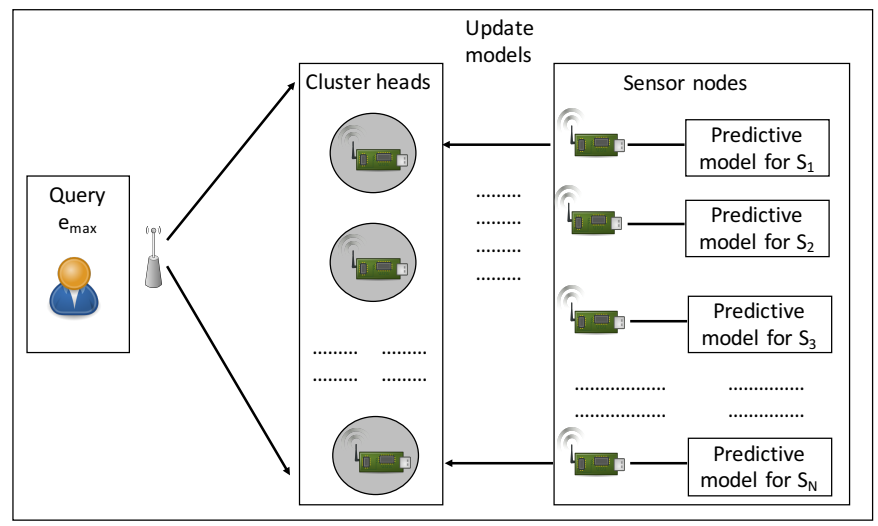

Fig. 2. The AM-DR model

before, using a combination scheme of two filters instead of using one provides an enhancement of both convergence and steady-state accuracy of the convex weight parameter for a better prediction of next data observation [12].

In equ. 4, we consider one of the adaptive filters $\left(w_{1}\right)$ is a fast filter while the other $\left(w_{2}\right)$ is slow in terms of their window size such that $w_{1}$ has a short-term observation memory based on using a relatively small fixed window size $w_{f}$ while the slow filter $w_{2}$ has a long-term observation memory based on an increasingly large window size $w_{s}$ [17] (Fig. 3 shows an example). It is worth mentioning that when $\lambda$ is near 1 , LMS coefficient is updated based on fast filter $w_{1}$ and when it is near 0 , the weight is updated based on the slow filter $w_{2}$.

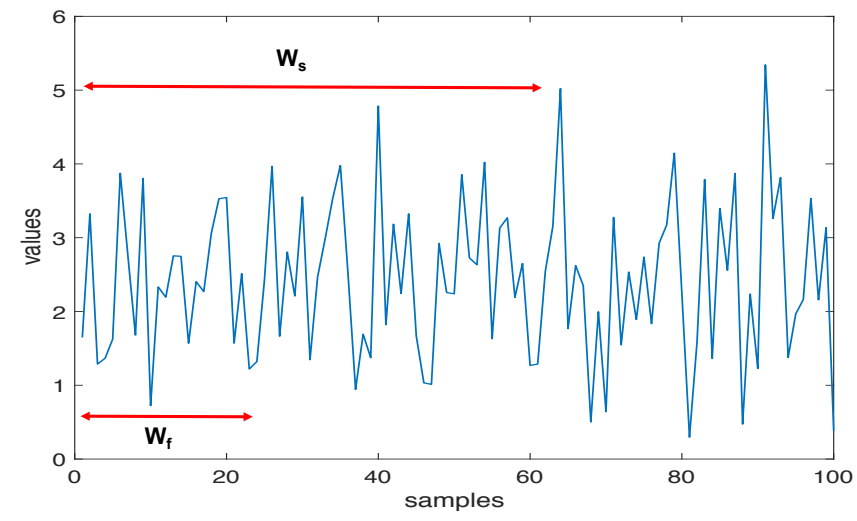

Fig. 3. Filter window sizes: a slow window with a size $w_{s}$ and a fast window with a size $w_{f}$

To capture the dynamic changes in sensor readings, a convex combination of two moving average filters (as mentioned above with two different window sizes $w_{f}$ and $w_{s}$ ) is used. The output of adaptive filters are $\hat{y}_{f}$ and $\hat{y}_{s}$ with a fixed window $w_{f}$ for fast filter and an increasing window $w_{s}$ for slow filter, respectively, such that $w_{f}<w_{s}$ and $\grave{t}$ is the current time index. 


$$
\begin{aligned}
& \hat{y}_{f}=\frac{1}{w_{f}} \sum_{t=\grave{t}-w_{f}}^{\grave{t}} x(t) \\
& \hat{y}_{s}=\frac{1}{w_{s}} \sum_{t=\grave{t}-w_{s}}^{\grave{t}} x(t)
\end{aligned}
$$

Similar to [11], the overall output for filters $\hat{y}(t)$ is a convex ${ }^{4}$ combination of the outputs of both filters mentioned above. A 5 combination of two filters $\hat{y}_{f}$ and $\hat{y}_{s}$ produces a single hybrid ${ }_{7}$ filter $\hat{y}(t)$ without any knowledge or assumption about input sensor readings $x(t)$. It is worth noting that the two filters 8 are running on parallel and their results feed into the mixing ${ }_{10}^{9}$ parameter $\lambda$ which produces a single overall output of the filter ${ }_{11}$ $\hat{y}(t)$.

$$
\hat{y}(t)=\lambda(t) \hat{y}_{f}+[1-\lambda(t)] \hat{y}_{s}
$$

where the mixing parameter $\lambda$ of their combination is adaptively updated in an online fashion that aims at minimising the error of overall filters $e(t)$ between the actual sensor reading $x(t)$ and overall output of both filters $\hat{y}(t)$.

$$
e(t)=[x(t)-\hat{y}(t)]
$$

AM-DR relies on the construction of an initial identical predictive model (i.e. during the initialisation mode) at both the $\mathrm{CH}$ and sensor node (as mentioned earlier). It is clear that the sensor node senses the actual readings; however, the $\mathrm{CH}$ relies on the constructed predictive model to predict the sensor's observation values. Since both $\mathrm{CH}$ and sensor node has started with the same initialisation, they can predict the next observation value (with an acceptable variation) simultaneously without a direction communication (i.e. during standalone mode). On the other hand, sensor node has to transmit only its sensed value $(x(t))$ if it deviates significantly ( $>$ a predefined threshold $\left.e_{\max }\right)$ from the predicted values $(\hat{y}(t))$ (i.e. during the normal mode). The details of these three modes are explained later in this section. It is worth mentioning that the error $e(t)$ is a key factor at the sensor node. More precisely, $\mathrm{CH}$ relies on using the predicted value as the current observation until it receives new sensed value from sensor nodes. Sensor node calculates the difference (i.e. $e(t)$ ) between its actual sensed value and the expected value (using the predictive model) and sends only the real/actual observations that deviate significantly from the actual observation to $\mathrm{CH}$.

The motivation of our proposed approach is to extract the best properties of the independent and decoupled fast $\hat{y}_{f}$ and slow $\hat{y}_{s}$ filters by assigning and updating $\lambda$ that is a combination of both filters at each time instance $t$ for minimising the error $e(t)$.

$$
\lambda(t+1)=\lambda(t)+\alpha e(t)\left[\hat{y}_{f}-\hat{y}_{s}\right]
$$

where $\alpha$ is the learning rate (i.e. step size) parameter. The

\begin{tabular}{|c|c|}
\hline Parameter & Definition \\
\hline$N$ & Total number of sensor Nodes \\
\hline$M$ & Total number of sink Nodes \\
\hline$i$ & Sensor index, $n=1,2, \cdots, N$ \\
\hline$T$ & Total time duration \\
\hline$t$ & Time index, $t=1,2, \cdots, T$ \\
\hline$x(t)$ & $\begin{array}{l}\text { data streams that are published by one of } N \text { sensors } \\
\text { at a time } t\end{array}$ \\
\hline$w_{f}$ & $\begin{array}{l}\text { A relatively small fixed window size for a short-term } \\
\text { observation memory based filter (i.e. fast filter) }\end{array}$ \\
\hline$w_{s}$ & $\begin{array}{l}\text { An increasingly large window size for a long-term } \\
\text { observation memory based on filter (i.e. slow filter) }\end{array}$ \\
\hline$x_{e}$ & $\begin{array}{l}\text { The available data streams either the predicted values } \\
\text { or actual values (i.e. based on normal/stand-alone } \\
\text { mode) }\end{array}$ \\
\hline$\alpha$ & Learning rate (i.e. step size) \\
\hline$\lambda(t)$ & $\begin{array}{l}\text { Mixing weight parameter of the combination of both } \\
\text { filters at time } t\end{array}$ \\
\hline start & The initialise of slow filter window \\
\hline$w$ & The difference between output filters $\hat{y}_{f}-\hat{y}_{s}$ \\
\hline$\hat{y}_{S}$ & $\begin{array}{l}\text { Output of slow filter (i.e. moving average with an } \\
\text { increasing window size } w_{S} \text { ) at time } t\end{array}$ \\
\hline$\hat{y}_{f}$ & $\begin{array}{l}\text { Output of fast filter (i.e. moving average with a fixed } \\
\text { window size } w_{f} \text { ) at time } t\end{array}$ \\
\hline$e(t)$ & $\begin{array}{l}\text { Error between the desired signal } x(t) \text { and overall } \\
\text { output of both filters } \hat{y}(t)\end{array}$ \\
\hline$\hat{y}(t)$ & A convex combination of the outputs of both filters \\
\hline$e_{\max }$ & $\begin{array}{l}\text { Maximum absolute deviation from actual observation } \\
\text { values (i.e. minimum accuracy) }\end{array}$ \\
\hline$A C K$ & $\begin{array}{l}\text { An acknowledgement scheme such that nodes should } \\
\text { send their reading (sensed) values even if their pre- } \\
\text { dictive models at BS or CH are updated in order to } \\
\text { identify alive and dead sensor nodes }\end{array}$ \\
\hline
\end{tabular}
learning rate influences the stability and the convergence of

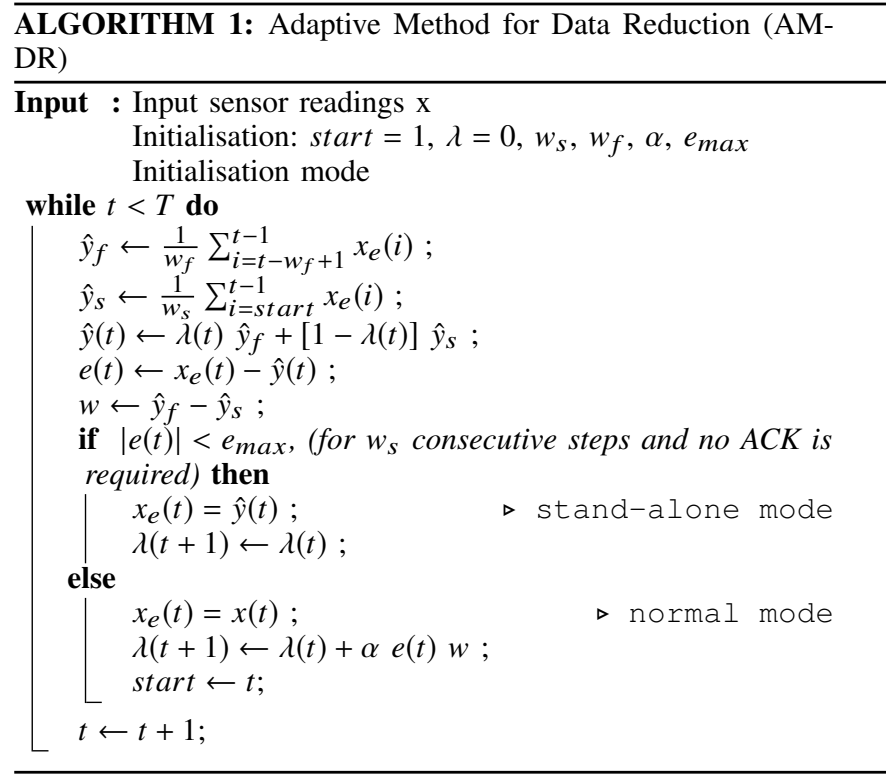

TABLE I

SUMMARY OF PARAMETERS

the model and it is known that LMS filters do not converge if $\alpha>1.0$ [28].

It is worth noting that LMS adaptive filter computes the estimation of $\hat{y}(t)$ of an input data value $x(t)$ at each time step $t$, as a linear combination of the last $N$ of sensory readings [2]. Thus, as a linear combination, the complexity of LMS is $\mathrm{O}(\mathrm{N})$ for each iteration. In AM-DR, for each iteration, we need to calculate the output of two filters, $\mathrm{O}\left(w_{s}+w_{f}\right)$, where $w_{s}$ and 
$w_{f}$ are the number of last samples for the slow and fast filters which can be approximately $O\left(w_{s}\right) ; w_{s}>w_{f}$.

The proposed algorithm (AM-DR) is summarised in Algorithm 1 and the definition of different parameters used in AM-DR are shown in Table I] Similar to [2], nodes have three main modes:

- initialisation mode: sensor nodes should send a certain amount of sensor readings (i.e. the first $w_{s}$ observations) at the beginning of running AM-DR to $\mathrm{CH}$ without making any predictions of sensor readings (i.e. before constructing prediction models). During this mode, the learning rate $\alpha$, window size for fast filter $w_{f}$, ACK (i.e. a span of time at which acknowledgement scheme should be activated) and $e_{\max }$ (i.e. a maximum absolute deviation based on a user-defined quality bounds) have to be initialised at both of sensor and $\mathrm{CH}$ nodes with same values (Fig. 2). After this mode, the AM-DR model is executed at both the $\mathrm{CH}$ and sensor nodes simultaneously, and they will switch between the normal and stand-alone modes.

- normal mode: a sensor node executes this mode when

- its predictive model at $\mathrm{CH}$ does not provide a good approximation of its following sensor readings according to a user-defined quality. More precisely, a sensor node is in a normal mode when the difference between the actual sensor data value $x(t)$ and predicted value $\hat{y}(t)$ (i.e. the convex combination of the outputs of both filters) is not within a user-defined quality (i.e. $\left.>e_{\text {max }}\right)$. In such a case, the mixing weight parameter of the convex combination of both filters $(\lambda)$ has to be updated. If there is a number of $w_{s}$ consecutive steps such that the prediction error $e(t)<\left|e_{\text {max }}\right|$, the node should switch to stand-alone mode because it has now enough sensor observations to predict the upcoming measurements.

- it requires sending an acknowledgement to $\mathrm{CH}$. The sensor nodes may not have enough energy to transmit all the sensor observations kept in their memory or failed for some other reasons. In such cases, the sensor node has a failure or is dead. To this end, failed or dead nodes should be identified, so $\mathrm{CH}$ stops predicting their coming values (for saving more energy) until their batteries are replaced. To address this problem, a node has to send only one actual observation value if it has not transmitted any readings to $\mathrm{CH}$ for a defined ACK time.

- stand-alone mode: the node works in a stand-alone mode when the prediction model is good enough to predict upcoming measurement with a deviation of $<\left|e_{\max }\right|$. To this end, the convex combination filter weight $(\lambda)$ does not need to be updated (i.e. the error $e_{t}=0$ ).

It is worth noting that since the $w_{s}$ is a filter with an increasing window, it has to be reinitialised (i.e. to get a fresh start) with the current $t$ value $($ start $=t$ ) only during the normal mode. This is because the combination filter weight $(\lambda)$ has to be updated during the normal mode. Although we have explained our AM-DR in a clustered network (Figs. 1 and 2,
AM-DR can also be employed in other network topologies (e.g. star, tree) between each pair of nodes that are directly connected.

\section{B. Energy model}

We have modified first order radio model [9, 10] to demonstrate radio characteristics in terms of energy dissipation of transmitting, receiving and processing modes for each sensor node. We adopt using the assumptions about the radio characteristics from the model in [9, 10] as follows. We assume that each sensor node has an initial energy $E_{o}=0.5$ (in Joules $(\mathbf{J})$ ). The energy $E$ for each sensor node decreases as the energy of node depletes during transmission, receiving and processing. We also assume that radio dissipates for each bit is $50 n J$ for transmitter and receiver electronics $\left(E_{\text {ele }}=50 \mathrm{~nJ} / \mathrm{bit}\right)$ and amplifier energy is $\epsilon_{f s}=10 \mathrm{pJ} / \mathrm{bit} / \mathrm{m}^{2}$ or $\epsilon_{m p}=0.0013 \mathrm{pJ} / \mathrm{bit} / \mathrm{m}^{4}$ for free space (fs) and multipath (mp) models, respectively. The selection between fs and $\mathrm{mp}$ model is based on the distance $d$ between the sender and the receiver such that fs model is used if $d<d_{t}$ ( $d_{t}$ is the maximum distance threshold between the sender and receiver), otherwise mp model is used. We can summarise the energy model for each sensor node (i.e. non- $\mathrm{CH}$ ) as follows:

$$
E_{T x}=\left(l \times E_{\text {ele }}\right)+E_{\text {amp }}
$$

where $E_{T x}$ is the energy dissipation for each sensor node for sending a message with a length $l$ (in bits) to $\mathrm{CH}$. $E_{\text {ele }}$ is the energy dissipation per bit for running the transmitter/receiver circuit. $E_{a m p}$ is the amplifier energy for transmission, and that is based on the distance $d$ between the receiver (i.e. $\mathrm{CH}$ ) and the sender (i.e. sensor (non-CH) node) such that:

$$
E_{a m p}= \begin{cases}l \times \epsilon_{f s} \times d^{2}, & \text { if } d<d_{t} \\ l \times \epsilon_{m p} \times d^{4}, & \text { if } d \geq d_{t}\end{cases}
$$

On the other hand, $E_{D A}=5 \mathrm{~nJ} /$ bit is the required energy at $\mathrm{CH}$ for data processing (e.g. data aggregation) of the received sensor readings (from its cluster members) before forwarding it to $\mathrm{BS}$. We mean by cluster members are (non- $\mathrm{CH}$ ) nodes that connect and transmit their data to $\mathrm{CH}$ (as shown in Fig. 1).

We assume that the size of the message that each node transmits to its $\mathrm{CH}$ that is located at a $d$ distance and the size of the aggregated message that $\mathrm{CH}$ sends to $\mathrm{BS}$ is $l=4000$ bits. In such a case, the energy cost for $\mathrm{CH}$ for transmitting an aggregated message to $\mathrm{BS}$ can be formulated as

$$
E_{T x}=l \times\left(E_{\text {ele }}+E_{D A}\right)+E_{a m p}
$$

where $E_{T x}$ is the energy dissipation for each $\mathrm{CH}$ to process and transmit a message to $\mathrm{BS} . E_{D A}$ is the energy cost of aggregation per bit. The aggregation cost is the cost of $\mathrm{CH}$ to aggregate data received from (non- $\mathrm{CH}$ ) nodes in an IoT network. $E_{a m p}$ is the same as mentioned before. For each $\mathrm{CH}$, the energy cost for receiving sensor readings from the cluster members before processing and transmitting them to BS is $E_{R x}$ such that 


$$
E_{R x}=l E_{\text {ele }}
$$

where $l$ is the total number of bits, a $\mathrm{CH}$ receives. We assume the size of the messages sent from the nodes to $\mathrm{CH}$ and from the $\mathrm{CH}$ to the $\mathrm{BS}$ is constant and equal to 4000 bits. However, if we consider that each cluster member transmits a message $l$ (with a different number of bits), $E_{R x}$ for each $\mathrm{CH}$ can be formulated as follows:

$$
E_{R x}=E_{\text {ele }} \sum_{i=1}^{N} l_{i}
$$

where $i(i=1,2, \cdots, N)$ is a sensor index that is associated with a given $\mathrm{CH}$ and $l_{i}$ is the message's length (in bits) that a sensor $i$ transmits to its $\mathrm{CH}$ and $N$ is the number of cluster members for the $\mathrm{CH}$.

It is worth noting that equ. 11 is the energy cost for each sensor node (i.e. non- $\mathrm{CH}$ ) to transmit a message with a $l$-bits to $\mathrm{CH}$, equ. 14 is the energy cost for each $\mathrm{CH}$ to receive sensor readings and equ. 13 is the energy cost of processing and forwarding the received readings to BS.

Our AM-DR model is based on the assumption that the required energy for radio transmissions in WSNs is some order of magnitude higher than the energy required for local processing at sensor nodes (as discussed and shown in [22, 29. 30, 31]). We consider that each cluster member (i.e. sensor node) depletes $E_{D A}$ (per bit) as a local processing energy cost for running AM-DR for predicting its coming sensor reading such that $E_{D A}=5 n J / b i t$ with an assumption that each predicted value requires a $l$-bits. Executing AM-DR at each $\mathrm{CH}$ requires $N$ order of magnitude higher than sensor node (i.e. non- $\mathrm{CH}$ ), where $N$ is the number of the cluster members of a given $\mathrm{CH}$. This is because each $\mathrm{CH}$ requires executing an instance of AM-DR for each individual node of its cluster members to predict the coming sensor readings for each sensor node. To this end, the energy dissipation for executing AM-DR $\left(E_{P x}\right)$ for $\mathrm{CH}$ and non-CH can be formulated as follows:

$$
E_{P x}= \begin{cases}l \times E_{D A} & \text { for non-CH } \\ l \times N \times E_{D A} & \text { for } \mathrm{CH}\end{cases}
$$

Overall, the total energy dissipation for $\mathrm{CH}$ and sensor nodes including the local processing for executing AM-DR strategy is presented in equ. 17, while the total energy dissipation without relying on the constructed prediction model within AM-DR strategy is presented in equ. 18. In equ. 17. using AM-DR predictive models, $\mathrm{CH}$ requires a local processing for AM-DR predictive models for $N$ cluster members with a cost of $E_{P_{x}}$ and transmitting an aggregated message to BS with a cost of $E_{T_{x}}$. On the other hand, without using AMDR models, $\mathrm{CH}$ receives every sensing values from cluster members $\left(E_{R_{x}}\right)$ and transmit an aggregated message to BS $E_{T_{x}}$, while non-CH transmits every value with a cost of $E_{T_{x}}$. To this end, we can infer equ. 19 (using equ. 11. equ. 16 . equ. 13 and equ. 17) and equ. 20 (using equ. 11 equ. 13. equ. 14 and equ. 18 .

$$
\begin{gathered}
E= \begin{cases}E_{P_{x}}+E_{T_{x}} & \text { for } \mathrm{CH} \\
E_{P_{x}} & \text { for non-CH }\end{cases} \\
E= \begin{cases}E_{R_{x}}+E_{T_{x}} & \text { for } \mathrm{CH} \\
E_{T_{x}} & \text { for non-CH }\end{cases} \\
E= \begin{cases}l \times N \times E_{D A}+l \times\left(E_{\text {ele }}+E_{D A}\right)+E_{\text {amp }} & \text { for } \mathrm{CH} \\
l \times E_{D A} & \text { for non-CH }\end{cases} \\
E= \begin{cases}l E_{\text {ele }}+l \times\left(E_{\text {ele }}+E_{D A}\right)+E_{\text {amp }} & \text { for } \mathrm{CH} \\
l \times\left(E_{\text {ele }}+E_{D A}\right)+E_{\text {amp }} & \text { for non-CH }\end{cases}
\end{gathered}
$$

We have selected LEACH cluster-based protocol to integrate our AM-DR and its energy model for quantifying the required energy of each individual sensor node and demonstrating how AM-DR can effectively prolong the network lifetime. Our motivation for this selection is that LEACH is one of the most appropriate protocols for monitoring scenarios (which is our target scenarios) in sensor networks [32].

It is worth noting that sensor nodes should have the same initial energy $E_{o}$ (that was explained before) in LEACH protocol. Interested readers can refer to the review by Afsar and Tayarani-N [33] for a detailed discussion about LEACH.

\section{EXPERIMENTAL EVALUATION}

This section explains the real-world datasets used in our evaluation, the parameter settings and reproducibility of our results and the evaluation criteria. It also includes analysis and discussion of our simulation results.

\section{A. Dataset}

We give a short explanation of the datasets that are used to evaluate our approach AM-DR compared to the baseline approach discussed in [2]. We have conducted our experiments on three real-world datasets including a dataset with drifts.

Our first experiments are based on real-world weather data (dataset 1, for brevity) that is available at: (http://db.lcs. mit.edu/labdata/labdata.html). The dataset is collected by 54 Mica2Dot sensors 1 with weather boards. Each sensor has the following parameters: temperature, humidity, light, voltage values, data and time at which a sensor reading is obtained and a sensor identifier (i.e. moteid). A clustered view for Mica2Dot sensors with weather boards at Intel Berkeley Research lab is shown in Fig. 4 We conducted our experiments in our previous work in [6] on temperature values. In this paper, we use humidity values.

Our second real-world dataset (dataset 2, for brevity) is appliances energy prediction dataset [34]. The data is available at: (http://archive.ics.uci.edu/ml/datasets/Appliances+energy+ prediction). The data was collected in different rooms at a house (e.g. kitchen, living, laundry, office) for monitoring

\footnotetext{
${ }^{1}$ http://www.willow.co.uk/html/mpr5x0__mica2dot_series.php
} 
temperature and humidity conditions with a ZigBee WSN. We have selected to run experiments on temperature sensors in the kitchen area.

Our third real-world dataset (dataset 3, for brevity) is air quality dataset [35]. The data is available at: (http://archive. ics.uci.edu/ml/datasets/Air+quality). The data was collected by chemical sensors for monitoring air quality in a polluted area at road level within an Italian city. The data contains values of concentrations for CO (in $\mathrm{mg} / \mathrm{m}^{3}$ ), Non Metanic Hydrocarbons (in $\mu g / m^{3}$ ), benzene (in $p p m$ i.e. part per million), Nitrogen Oxides $\left(N O_{x}\right)$ (in $p p b$ i.e. part per billion) and Nitrogen Dioxide $\left(\mathrm{NO}_{2}\right)$ (in $\left.\mu \mathrm{g} / \mathrm{m}^{3}\right)$. We have removed the missing values (i.e. missing values are tagged with -200 value). As described in [35], the dataset has sensor drifts due to distribution changes and/or sensor ageing effects. We are interested in testing our approach on benzene concentration for urban pollution monitoring scenarios to show how AMDR can adapt with drifts by utilising two adaptive filters with differing window sizes.

The advantage of the adaptive combination of two filters (i.e. fast and slow) is that the fast filter has a higher tracking capability that follows the abrupt changes well and the slow filter has a better steady-state that minimises the detection error.

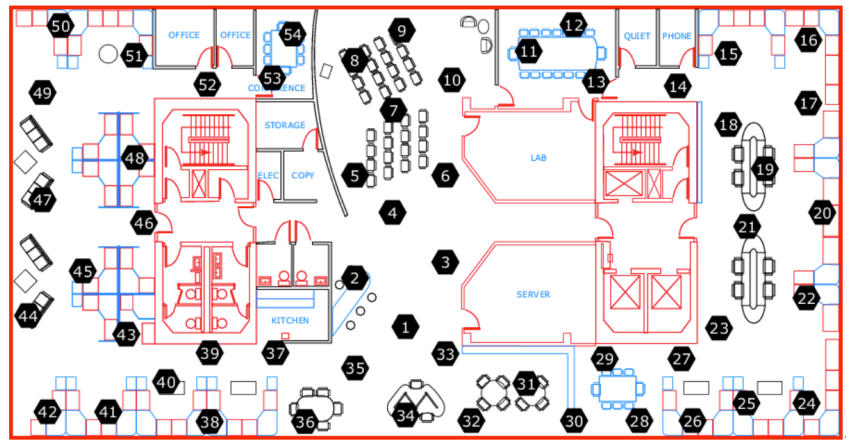

Fig. 4. A clustered view for Mica2Dot sensors with weather boards at Intel Berkeley Research lab

\section{B. Parameter settings and reproducibility}

We have two sets of experiments. The first set is to compare our approach with different real-world datasets against the baseline approach in [2]. For the first set of experiments, we evaluate the performance of our method (AM-DR) against the baseline approach on three different datasets that are explained previously. We have used $\left(N=5, \mu=10^{-5}\right)$ for the baseline approach (as reported in [2]). On the other hand, the following are the specific default values that we have used for each of the parameters in our approach $\left(w_{f}=5, w_{s}=10, \alpha=1.0 e-007\right)$.

During empirical experimentation, we have noticed that $w_{s}$ should be doubled the value of $w_{f}$. Since $N$ parameter value of the baseline approach in [2] is 5, we have also used $w_{f}=5$ and $w_{s}=10$.

For the second set of experiments, we integrate our AMDR and its energy model that is described in Section IV] into LEACH code that is available at: (http://csr.bu.edu/sep/). In this case, we use the first real-world dataset to quantify the energy requirement of each individual sensor node.

To ensure the reproducibility of our results, we have made the code and dataset of our implementation and baseline available and have also provided details of a configurable experimental set-up at: (http://github.com/YasminFathy/ AMDREnergyModel).

\section{Evaluation criteria}

Transmitting data between sensor nodes and cluster heads consumes higher energy than data sensing [2, 6]. To this end, large energy gains can be achieved in the IoT networks by executing AM-DR at both $\mathrm{CH}$ and sensor nodes, thereby reducing the number of data transmission between sensor nodes and their CHs. We have two sets of experiments whose evaluation criteria are different. For the first set of experiments (use-case I, for brevity), our evaluation criteria are as follows:

- Percentage of transmitted data: to evaluate the communication overhead between nodes by assessing how much a sensor node should transmit its sensor readings to $\mathrm{CH}$ without utilising/depending on the prediction model.

- Prediction error: defined as the difference between the actual sensor and predicted readings

For the second set of experiments (use-case II, for brevity), we evaluate our energy model within the LEACH protocol. In such a case, our evaluation criteria are as follows:

- Number of alive nodes: the number of nodes in the network that remain alive for a long time.

- Stability period (SP): the time interval just before the first node is dead (i.e. run out of its energy) [36].

- Throughput (Tp): the rate of data (in Kilobits (Kbits)) sent from the sensor nodes to their $\mathrm{CH}$.

It is worth noting that the higher number of nodes that remain alive, as well as, the higher the values of $\mathrm{Tp}$ and $\mathrm{SP}$, the more effective our approach is performing within a period $T$. More precisely, running the network for time $T$, we show that utilising our approach within the network saves more energy for the entire network and consequently, prolonging the network lifetime.

\section{RESULTS AND DISCUSSION}

As discussed in the previous section, we have two cases for the experiments use-case I and use-case II. In use-case I, we have compared our algorithm with the state-of-the-art algorithm (baseline) in [2]. In use-case II, we have evaluated our energy model to quantify and evaluate how much energy dissipation can be reduced by integrating our approach into the LEACH protocol.

\section{A. Use-Case I}

Using dataset 1 , we have used data reported by the humidity values that are collected from motes $(30,49)$ between March 6 and 9. We have compared our AM-DR approach against the baseline approach [2].

Fig. 5 ( $a$ and $b$ ) shows the error of both approaches (i.e. with a requested data quality $98 \%$, i.e. $e_{\max }=2$ ). The red circle 
indicates the sensor readings that have to be transmitted to the $\mathrm{CH}$. The figures indicate that when the prediction error exceeds $\left|e_{\text {max }}\right|$, the sensor nodes transmit their readings to their $\mathrm{CH}$ (i.e. normal mode). As mentioned in Section IV-A that $w_{s}$ is an increasingly large window size that has to be reinitialised to get a fresh start only during the normal mode. In the normal mode, the sensor node transmits its actual observation to $\mathrm{CH}$. To this end, the prediction error starts to decrease and to fully depend on the predictive mode later (i.e. switch to stand-alone mode), the sensor node is required to send $w_{s}$ sensed values such that there are enough observations to make a prediction and switches to a stand-alone mode.

Fig. 6 ( $a$ and b) demonstrates the results of AM-DR and baseline approaches. They demonstrate the difference between actual and predicted sensor readings. AM-DR achieves $93 \%$ communication reduction, and the baseline achieves $84.3 \%$ while maintaining $98 \%$ data quality; a small deviation of 2 degree between the actual and predicted sensor readings. We have conducted the same experiments on collected data from mote 49 , and we have quite a similar conclusion on how AMDR outperforms the baseline (Please refer to Appendix A-A for more details about these experiments).

Fig. 7( $(a$ and $b$ ) shows the percentage of the transmitted data between sensor nodes (i.e. motes 30 and 49) and their $\mathrm{CH}$ with different user-defined data quality. For instance, the baseline requires transmitting up to $15 \%$ of sensor data to obtain up to $4 \%$ data quality while our approach requires only about $4 \%$ to retain the same quality for mote 30 . Similarly, our method requires transmitting up to $3 \%$ of readings while the baseline requires up to $14 \%$ of data to be transmitted from sensor nodes to $\mathrm{CH}$ for mote 49 .

It is clear that as the requested quality decreases (i.e. higher values of $e_{\max }$ ), the communication between sensor nodes and $\mathrm{CH}$ reduces. This is because as the data quality gets lower, a higher difference between the prediction and actual sensor readings is accepted and consequently, sensor nodes do not require to transmit an updated value of their immediate readings. Overall, our approach on this dataset can achieve a communication reduction up to 10 order of magnitude higher than the baseline approach while retaining the same quality. To this end, AM-DR mediates successfully between a userdefined quality and energy efficiency. It is worth noting that we show only the samples between 1540 and 1640 (for better visualisation); however, we conduct our experiments on the entire collected data for humidity values (4000 samples), and we report the communication reduction for the 4000 samples.

It is worth noting that mote 30 and 49 in dataset 1 have more spiky data than dataset 2 . We would expect that our approach has a higher communication reduction in dataset 1 than dataset 2 comparing to the baseline approach. The main advantage of our approach lies in using two adaptive filters with different sizes such that the dynamicity in sensor readings is captured.

Using dataset 2, Fig. 8 ( $a$ and b) demonstrates the results of AM-DR and baseline approaches. They demonstrate the difference between actual and predicted sensor readings. Fig. 8 (a) also depicts how predicted values deviate significantly from actual values in the baseline case that results at increasing the number of transmissions compared to AM-DR (more details check Fig. 14 in Appendix A-B. On the other hand, Fig. 8 (b) shows when the prediction error exceeds $e_{\max }=|2|$, the sensor node sends its observation to its $\mathrm{CH}$ (indicated by a red circle). As soon as the prediction error decreases at least $w_{s}$ times such that there are enough readings to make a prediction, the sensor node switches to a stand-alone mode.

Fig. 9 (a) depicts the percentage of transmitted data from a sensor node to its $\mathrm{CH}$ (Please refer to Appendix $\mathrm{A}-\mathrm{B}$ for more details about the prediction error of temperature sensor readings for this dataset). Our experiments as shown in Fig. 9 (a), show that AM-DR requires only to transmit 5\% while the baseline requires $9 \%$ of sensor readings to be transmitted such that the difference between the actual and predicted observations is only $1^{\circ} \mathrm{C}$ degree. When the acceptable difference between the actual and predicted readings is $2^{\circ} \mathrm{C}$ degree (based on application requirements), AM-DR outperforms the baseline such that the former requires $1 \%$ of the time to communicate with $\mathrm{CH}$ and the latter requires 5\%. Although, we have expected that the data transmission of our approach in dataset 1 will be higher than the data transmission in dataset 2, AM-DR reduces data transmission to $93 \%$ and $99 \%$ compared to the baseline that requires $84 \%$ and $95 \%$ for the same userdefined data quality $\left(e_{\max }=2\right)$ in dataset 1 and dataset 2 , respectively.

AM-DR can adapt well to the changes in the data as explained before. We are interested in studying the changes further. To this end, our last set of experiments are on sensor data with data drifts (using dataset 3).

Fig. 9 (b) shows the percentage of transmitted data from a sensor node to its $\mathrm{CH}$ of benzene concentration for air quality monitoring (Please refer to Appendix $\mathrm{A}-\mathrm{C}$ for more details about the difference between actual and predicted sensor readings and the prediction error for this dataset). It is worth mentioning that we have used $N=3$ for the baseline and consequently $w_{f}=3$ and $w_{s}=6$. We have selected a small window size because the data has drifted over time and having a small window size enables capturing the changes in the readings. We have also selected different quality values such that $e_{\text {max }}=[0,0.03]$ (in $p p m$ ). Our selection for this range of values is due to the fact that an Air Quality Standard (AQS) 2 for benzene is $5 \mathrm{ppb}$ which is $0.005 \mathrm{ppm}$. Furthermore, the benzene concentration has a limit value of $10 \mu \mathrm{g} / \mathrm{m}^{3}(0.01$ ppm) according to the European Community (EC 2000) [37]. Benzene can cause harmful effects on a human body (e.g. reduce red blood cells and affect the immune system). It can also cause acute myeloid leukaemia (AML) [38]. So, it is essential to monitor benzene concentration such that citizens can be alarmed to avoid areas with high benzene concentration levels.

Fig. 9 (b) depicts that our AM-DR can adapt very well to data drifts. It is clear that when a user-defined quality is only $0.01 p p b$ from the actual readings (i.e. $e_{\max }=0.01$ ), AM-DR requires to transmit only $21 \%$ while the baseline requires $52 \%$. This shows how our approach adapts well to dynamic changes while guaranteeing the requested data quality. Moreover, AMDR capabilities achieve an adequate trade-off between data

${ }^{2}$ https://uk-air.defra.gov.uk/assets/documents/reports/empire/benz/bench3.html 


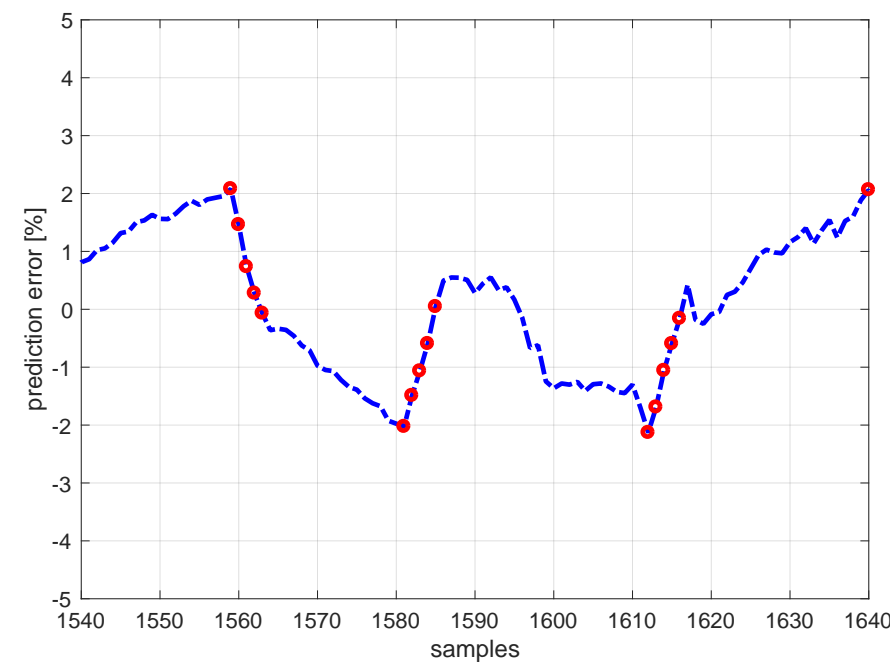

(a) Basline

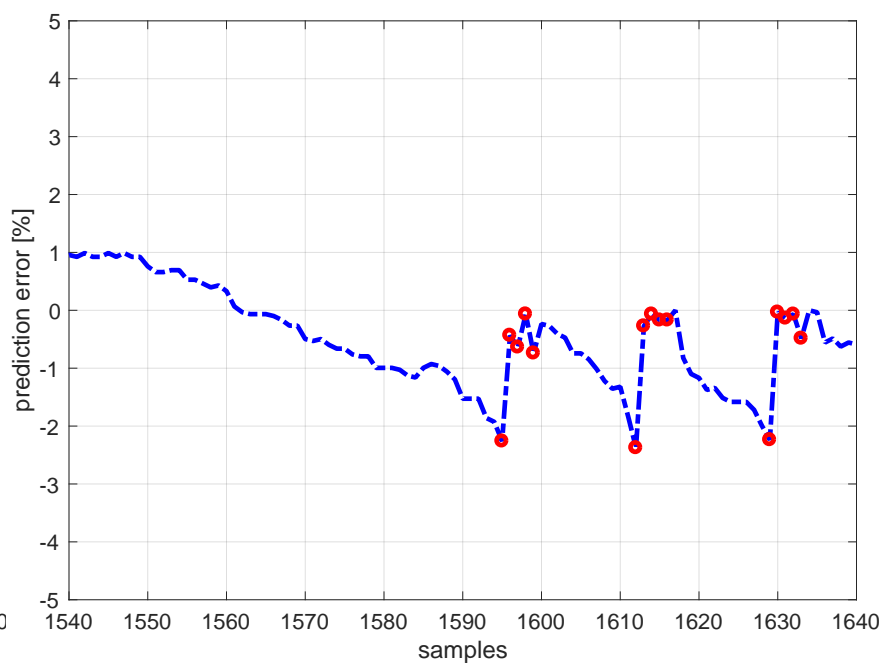

(b) AM-DR

Fig. 5. Dataset 1-Baseline vs AM-DR: prediction error of mote 30 with $e_{\max }=2$

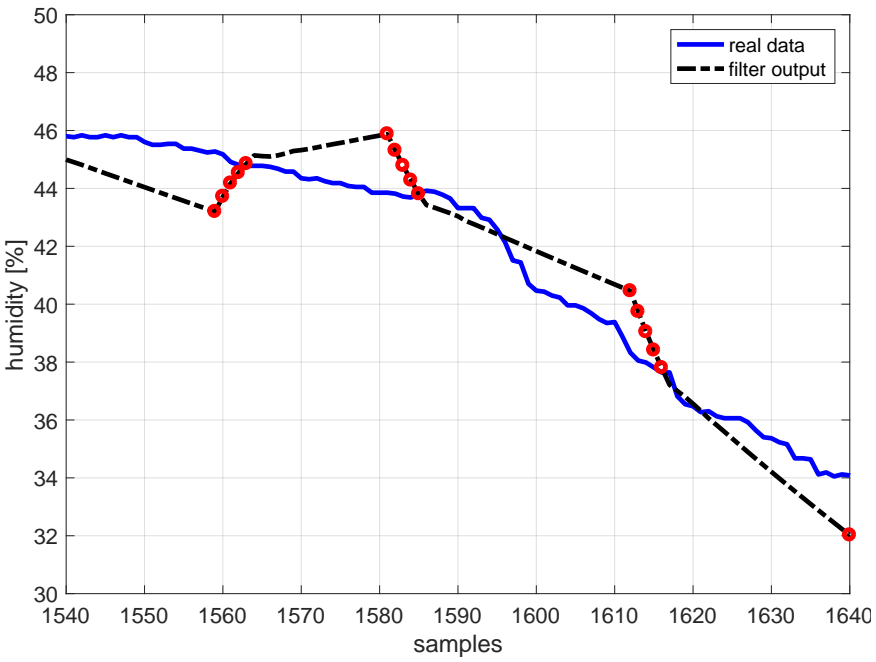

(a) Basline

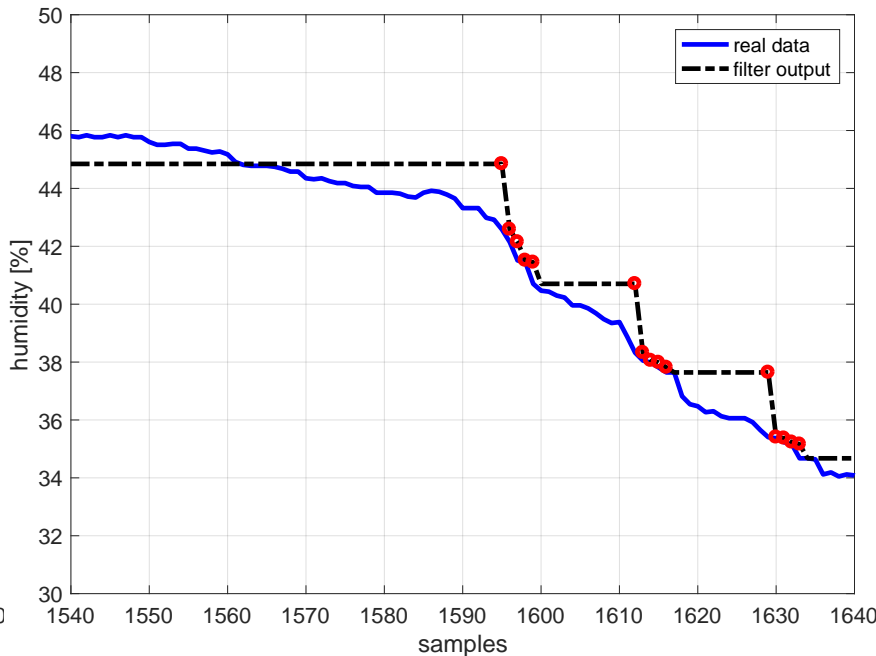

(b) AM-DR

Fig. 6. Dataset 1-Baseline vs AM-DR: real and predicted sensor readings of mote 30 with $e_{\max }=2$

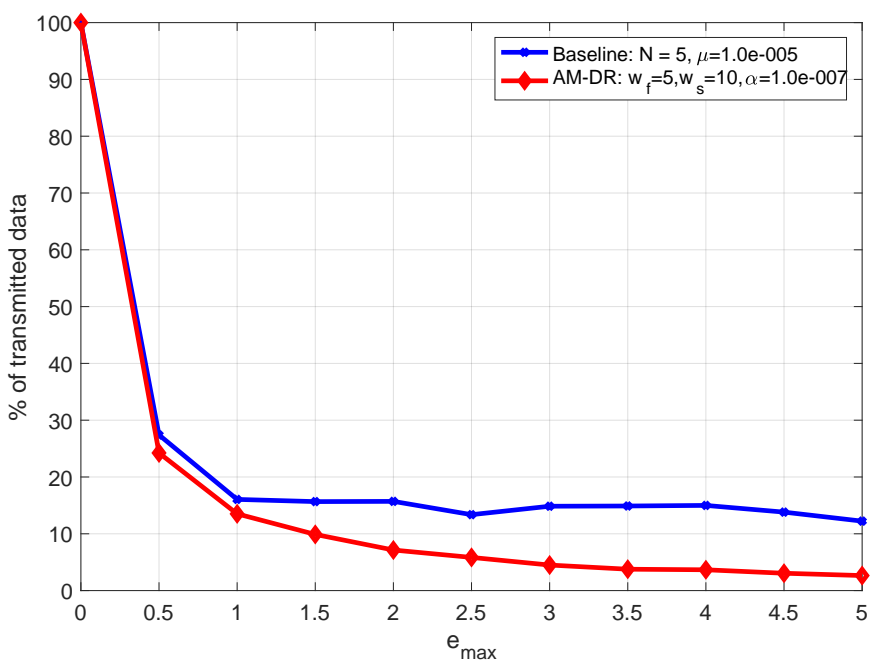

(a) mote 30

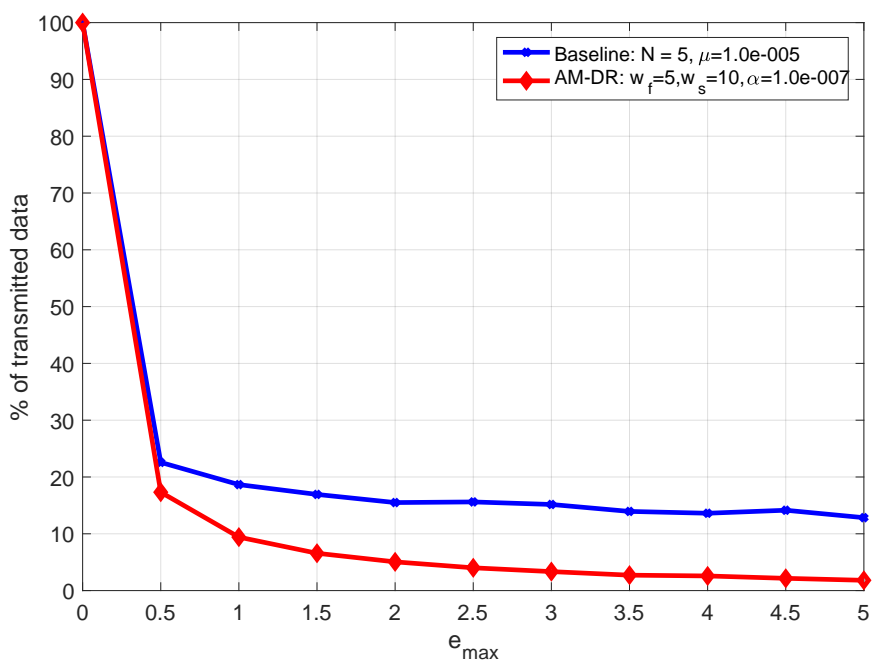

(b) mote 49

Fig. 7. Dataset 1-Baseline vs AM-DR: percentage of transmitted data by motes (30 and 49) with different requested data quality up to $95 \%$ 
quality for prediction and communication overhead such that a fine granular data that is transmitted from a sensor node enables original observation data to be reconstructed within some user-defined quality boundary at $\mathrm{CH}$.

It is worth noting that the percentage of transmitted data is $100 \%$ when $e_{\max }=0$ because this means that the userdefined deviation of predicted values from actual values is zero such that a sensor node has to transmit its immediate sensor readings to $\mathrm{CH}$.

\section{B. Use-Case II}

Having sensor nodes that are out of battery in WSNs is a potential problem that affects the reliability and lifetime of the system. To this end, we have integrated AM-DR energy model (as explained previously in Section IV-B into the LEACH energy model to show the effect of our AM-DR approach to prolong the network lifetime as well as reduce the number of transmissions while retaining a user-defined quality. We have conducted a set of experiments using dataset 1 . More precisely, we generate a WSN of 52 nodes (i.e. the number of sensor nodes in dataset 1) that are randomly distributed over a $100 \mathrm{~m} \times$ $100 \mathrm{~m}$ field where a base station is located at the centre of the field. Furthermore, each sensor node (non- $\mathrm{CH}$ ) constructs a predictive model, and a similar model is constructed at its $\mathrm{CH}$. As discussed in the previous Sections, sensor nodes transmit and communicate with its $\mathrm{CH}$ if the predicted readings deviate significantly from the actual readings.

Fig. 10 ( $a$ and $b$ ) shows the network lifetime in LEACH with and without AM-DR. Fig. 10 (a) depicts the number of alive nodes per round. It is clear that the first node dies at round 790. We observe in the same figure that utilising AMDR prolongs the time interval before the death of the first node (i.e. is referred to as a stability period) such that the first node dies after a higher significant number of rounds (i.e. at round 2551) compared to LEACH without AM-DR. On the other hand, AM-DR prolongs the network lifetime such that until round 4000, there is still around 6 nodes that are alive, while without utilising AM-DR, the last node dies at round 1916.

When the number of alive nodes is increased in the network such that a longer stability period exists, the average throughput should be higher. This is clear in Fig. 10 (b) where the network can transmit around 578 Kbits until round 1500 while LEACH takes some advantage of AM-DR such that network is able to transmit 609 Kbits until the same round (i.e. 1500). More precisely, the entire network transmits 578 Kbits without AM-DR and 1531 Kbits with AM-DR.

\section{Sensitivity analysis}

In AM-DR, data reduction, data quality and the trade-off between them can be affected by different parameters such as $e_{\text {max }}$ and window sizes $\left(w_{f}\right.$ and $w_{s}$ ).

We have considered different quality values (i.e. $e_{\text {max }}$ ) in our experiments. We also observe (as discussed before) that $e_{\max }$ has to be initialised according to application requirements. Furthermore, our approach can be applied in both critical and non-critical applications. For instance, in related healthcare monitoring applications (e.g. dataset 3 (benzene concentration)), accurate sensor observations are necessary. In such a case, a small deviation value is required (as shown in Section VI-A. On the other hand, some other environmental monitoring (i.e. temperature and humidity) applications can consume less energy (i.e. reduce the number of transmitted sensor readings) by allowing a reasonable deviation of predicted values compared to actual sensor readings. Moreover, in fire detection and warning systems, the readings (e.g. temperature) might have a sudden high value which is higher than the pre-defined threshold (i.e. deviation value $e_{\max }$ ), which, in turn, requires sensor nodes to transmit their immediate readings to $\mathrm{CH}$ and consequently, the event of fire will be detected.

We have also shown in our experiments that when there are dynamic changes (e.g. dataset 3 with drifts), the window size $w_{f}$ should be small enough so that the dynamic changes are captured. Consequently, the $w_{s}$ should also be smaller such that $w_{f}<w_{s}$.

We believe that data reduction in terms of the fraction of transmitted messages with and without using prediction models can be affected by the data quality level (i.e. $e_{\max }$ value). To study this further, Suppression ratio (SR) is evaluated with different values of $e_{\max }$ for the same dataset to have a robust conclusion about the trade-off between data reduction and quality.

SR is defined as the fraction of the number of transmitted messages that can be avoided such that the constructed prediction model is used by the total number of messages in the network. SR can be represented as follows:

$$
S R=\frac{\text { No. of messages generated with prediction }}{\text { Total no. of messages in the system }}
$$

SR enables measuring how much data transmission can be reduced with the presence of AM-DR strategy to its absence in the network. Fig. 11 shows the suppression ratio (in \%) for different $e_{\max }$ values. SR is zero when AM-DR is completely suppressed (i.e. does not affect). This results in increasing the number of transmissions. More precisely, when $e_{\max }$ is zero, sensor nodes have to transmit their readings to $\mathrm{CH}$ such that the transmission rate is $100 \%$ (as shown previously in Fig. 7 and Fig. 97 and consequently, SR ratio is 0 .

It is worth noting that the higher value of SR, the less suppression of utilising AM-DR (i.e. the more effect of AMDR) on reducing the number of data transmission between sensor nodes and their $\mathrm{CH}$ in the network. We have observed that having a less deviation value between predicted and actual readings (i.e. small values of $e_{\max }$ ), requires more messages to be transmitted between sensor nodes and their $\mathrm{CH}$, thereby having a lower SR (i.e. higher suppression of AM-DR). It is worth mentioning that we used the same value for each of the parameters $\left(w_{f}=5, w_{s}=10, \alpha=1.0 e-007\right)$. This also has been verified in our previous experiments (in Fig. 7 and Fig. 9p such that a small deviation requires a higher percentage of transmitted data compared to a higher deviation. Overall, the suppression ratio reflects the level of effect of AM-DR strategy on data reduction with respect to 


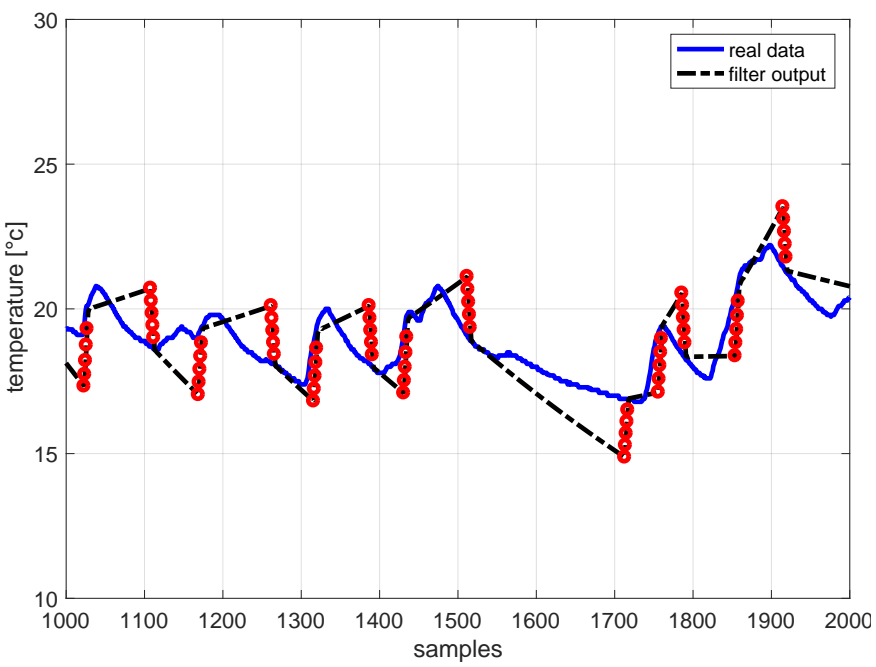

(a) Baseline

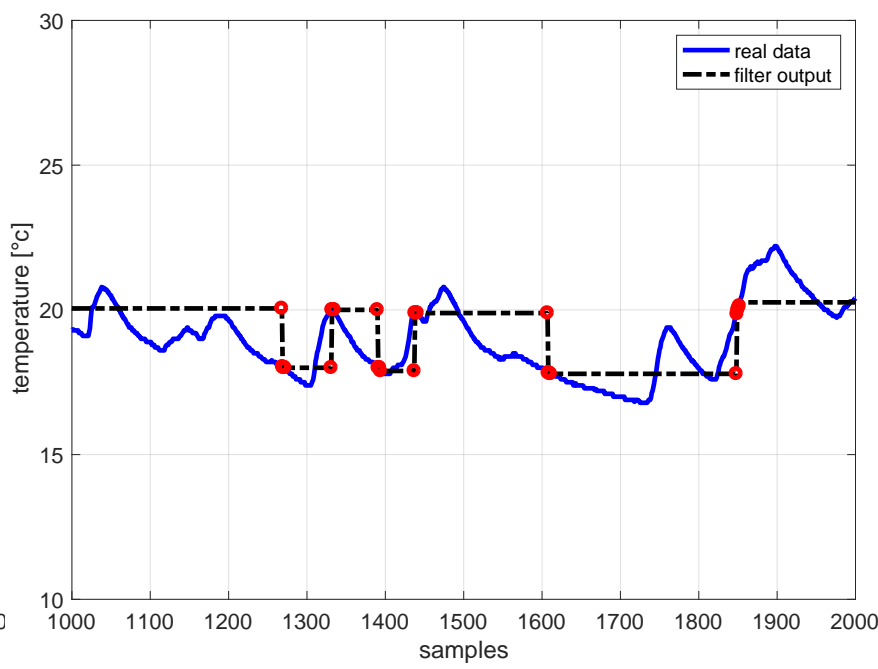

(b) AM-DR

Fig. 8. Dataset 2-Baseline vs AM-DR: real and predicted temperature sensor readings in kitchen with $e_{\max }=2$

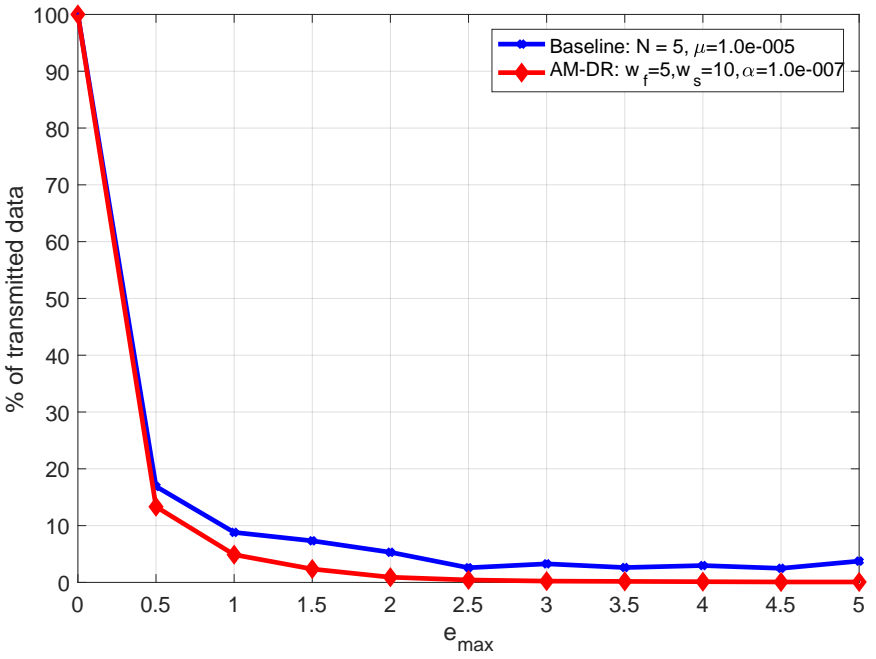

(a) Dataset 2-Energy data: temperature

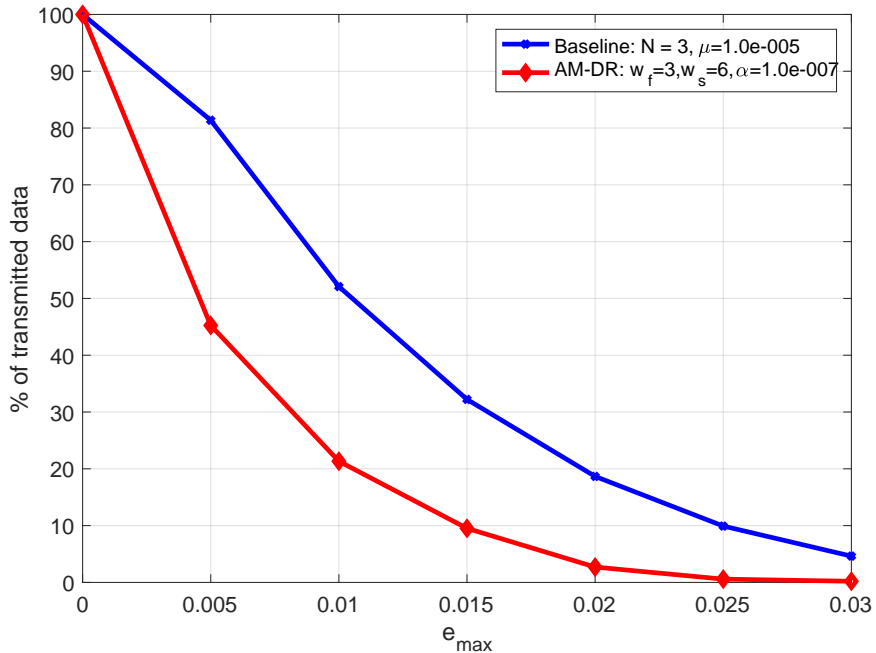

(b) Dataset 3-Air quality: benzene concentration

Fig. 9. Baseline vs AM-DR: percentage of transmitted data by a sensor node to $\mathrm{CH}$

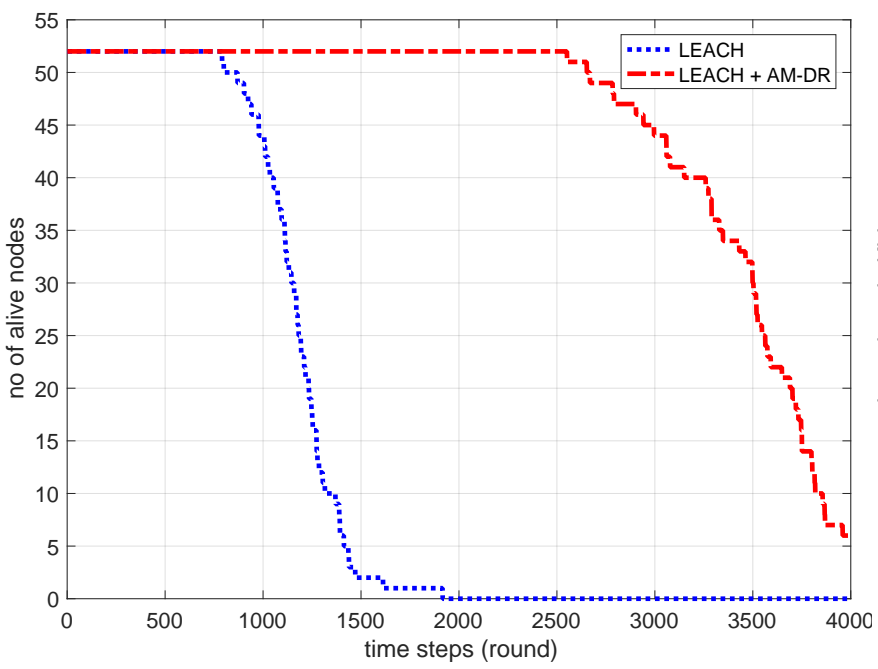

(a) Stability period

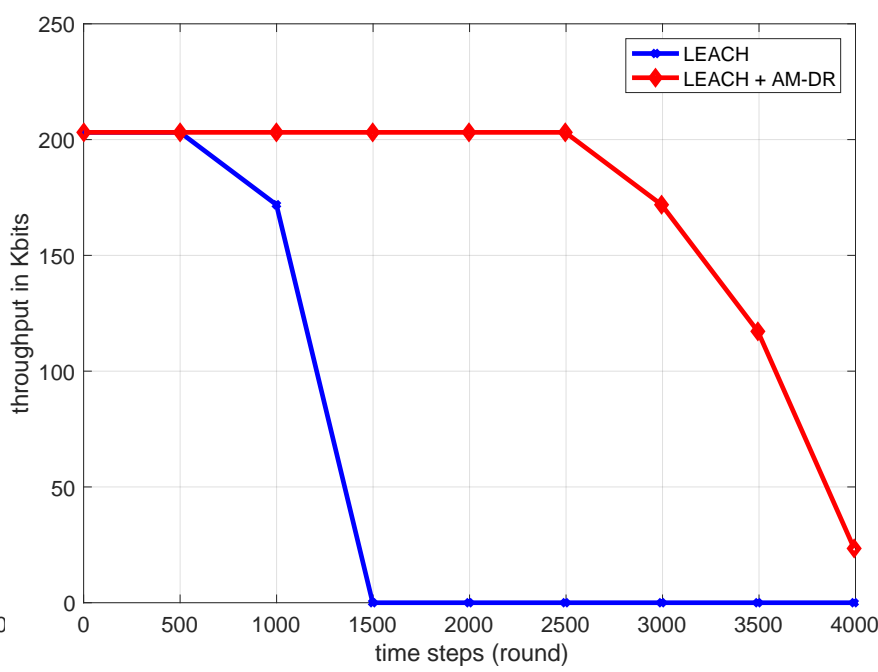

(b) Throughput in Kbits

Fig. 10. Network lifetime 
a user-defined quality boundary by relying on the constructed prediction-based models within AM-DR.

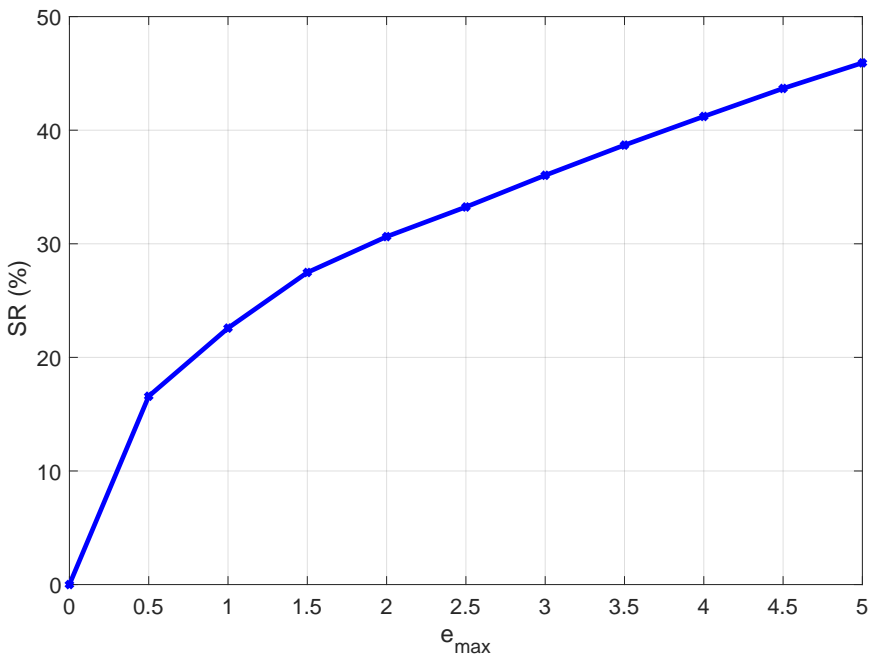

Fig. 11. Suppression ratio for AM-DR

\section{CONCLUSIONS AND FUTURE WORK}

We have discussed and demonstrated a novel Adaptive Method for Data Reduction (AM-DR) to reduce overall transmission and communication between sensor nodes in IoT networks. The proposed approach relies on exploiting finegrained sensor readings of real-world phenomena to reconstruct original sensed data within a user-defined accuracy. It employs dual prediction scheme based on a convex combination of two adaptive filters with differing window sizes for predicting upcoming measurements. Our approach requires no prior knowledge about the underlying data and allowing nodes to work independently in the network. It can also be employed in different network topologies (e.g. clustered, star, tree) within any pair of nodes that have a direct connection. To fully achieve the energy savings enabled by AM-DR, we have provided an energy cost model and have conducted two different sets of experiments.

Through our first set of experiments on different realworld datasets (use-case I), our algorithm has provided a high communication reduction. We have been able to achieve up to $90 \%$ (in dataset 1) communication reduction while maintaining a minimum accuracy of $\pm 2 \%$ for humidity sensor readings. In addition, communication overhead has been reduced up to $95 \%$ (in dataset 2) while maintaining a minimum accuracy of \pm 1 degree Celsius for temperature sensor readings and up to $90 \%$ (in dataset 3) while maintaining a minimum accuracy of $\pm 0.015 \mathrm{ppm}$ for benzene concentration readings.

Through our second set of experiments (use-case II), AMDR enables to prolong network lifetime such that the number of alive nodes has been increased in the network and that results at having a more extended stability period and higher throughput in the network. As discussed previously, the required energy for radio transmissions in sensor networks is some order of magnitude higher than the energy required for local processing at sensor nodes. We have also shown that a significant energy gain has been achieved by utilising AM-DR at both sensor and $\mathrm{CH}$ nodes, thereby requiring much fewer data to be transmitted between sensor nodes and their $\mathrm{CHs}$ while retaining a user-defined quality.

We are aware that there are many LEACH-like schemes and other protocols in the literature such as LEACH-B [39, 40], Stable Election Protocol (SEP) [36], among others. These protocols tend to focus on selecting cluster heads for extending the stability period of the network lifetime. Our approach focuses more on extending the stability period and network lifetime by reducing data transmission between sensor nodes. Although we have picked LEACH to integrate our energy model, our model can be incorporated into other protocols. AM-DR can be potentially applied in different context and scenarios including crowd-based applications.

Although our approach has shown better performance compared with the state-of-the-art and has also been able to mediate successfully between data quality and energy efficiency, the future work will focus on detecting event patterns at BS such that more complex queries can be answered. This will require adapting our approach to work on multi-dimensional sensor data such that each dimension might have different $e_{\max }$ value.

\section{ACKNOWLEDGMENT}

This work was partially supported by the EU Horizon 2020 IoTCrawler project under Grant No.: 779852 and the Care Research and Technology Centre at the UK Dementia Research Institute.

\section{REFERENCES}

[1] Y. Fathy, P. Barnaghi, and R. Tafazolli, "Large-Scale Indexing, Discovery, and Ranking for the Internet of Things (IoT)," ACM Comput. Surv., vol. 51, no. 2, pp. 29:1-29:53, Mar. 2018.

[2] S. Santini and K. Romer, "An adaptive strategy for quality-based data reduction in wireless sensor networks," in Proceedings of the 3rd international conference on networked sensing systems (INSS 2006), 2006, pp. 29-36.

[3] B. Babcock and C. Olston, "Distributed top-k monitoring," in Proceedings of the 2003 ACM SIGMOD international conference on Management of data. ACM, 2003, pp. 28-39.

[4] D. Ciuonzo and P. S. Rossi, "Quantizer design for generalized locally optimum detectors in wireless sensor networks," IEEE Wireless Communications Letters, vol. 7, no. 2, pp. 162-165, 2017.

[5] D. Ciuonzo and P. S. Rossi, "Distributed detection of a non-cooperative target via generalized locally-optimum approaches," Information Fusion, vol. 36, pp. 261-274, 2017.

[6] Y. Fathy, P. Barnaghi, and R. Tafazolli, "An Adaptive Method for Data Reduction in the Internet of Things," in "2018 IEEE 4th World Forum on Internet of Things (WF-IoT) (WF-IoT 2018)”. IEEE, 2018, pp. 729-735.

[7] N. Alinaghipour, H. Yousefi, M. H. Yeganeh, and A. Movaghar, "Long lifetime real-time routing in unreliable Wireless Sensor Networks," in Wireless Days (WD), 2011 IFIP. IEEE, 2011, pp. 1-6.

[8] G. M. Dias, B. Bellalta, and S. Oechsner, "A survey about predictionbased data reduction in wireless sensor networks," ACM Computing Surveys (CSUR), vol. 49, no. 3, p. 58, 2016.

[9] W. R. Heinzelman, A. Chandrakasan, and H. Balakrishnan, "Energyefficient communication protocol for wireless microsensor networks," in System sciences, 2000. Proceedings of the 33rd annual Hawaii international conference on. IEEE, 2000, pp. 10-pp.

[10] W. B. Heinzelman, A. P. Chandrakasan, and H. Balakrishnan, "An application-specific protocol architecture for wireless microsensor networks," IEEE Transactions on wireless communications, vol. 1, no. 4, pp. 660-670, 2002.

[11] M. Martinez-Ramon, J. Arenas-Garcia, A. Navia-Vázquez, and A. R. Figueiras-Vidal, "An adaptive combination of adaptive filters for plant 
identification," in Digital Signal Processing, 2002. DSP 2002. 2002 14th International Conference on, vol. 2. IEEE, 2002, pp. 1195-1198.

[12] J. Arenas-García, A. R. Figueiras-Vidal, and A. H. Sayed, "Steady state performance of convex combinations of adaptive filters," in Acoustics, Speech, and Signal Processing, 2005. Proceedings.(ICASSP'05). IEEE International Conference on, vol. 4. IEEE, 2005, pp. iv-33.

[13] Y. Zhang and J. A. Chambers, "Convex combination of adaptive filters for a variable tap-length LMS algorithm," IEEE Signal Processing Letters, vol. 13, no. 10, pp. 628-631, 2006.

[14] J. Arenas-Garcia, A. R. Figueiras-Vidal, and A. H. Sayed, "Mean-square performance of a convex combination of two adaptive filters," IEEE transactions on signal processing, vol. 54, no. 3, pp. 1078-1090, 2006.

[15] R. K. Martin, W. A. Sethares, R. C. Williamson, and C. R. Johnson, "Exploiting sparsity in adaptive filters," IEEE Transactions on Signal Processing, vol. 50, no. 8, pp. 1883-1894, 2002.

[16] D. Mandic, P. Vayanos, C. Boukis, B. Jelfs, S. L. Goh, T. Gautama, and T. Rutkowski, "Collaborative adaptive learning using hybrid filters," in Acoustics, Speech and Signal Processing, 2007. ICASSP 2007. IEEE International Conference on, vol. 3. IEEE, 2007, pp. III-921.

[17] D. Kalus, M. Muma, and A. M. Zoubir, "Distributed robust change point detection for autoregressive processes with an application to distributed voice activity detection," in Acoustics, Speech and Signal Processing (ICASSP), 2015 IEEE International Conference on. IEEE, 2015, pp. 3906-3910.

[18] B. L. R. Stojkoska and K. V. Trivodaliev, "A review of Internet of Things for smart home: Challenges and solutions," Journal of Cleaner Production, vol. 140, pp. 1454-1464, 2017.

[19] A. Deshpande, C. Guestrin, S. R. Madden, J. M. Hellerstein, and W. Hong, "Model-driven data acquisition in sensor networks," in Proceedings of the Thirtieth international conference on Very large data bases-Volume 30. VLDB Endowment, 2004, pp. 588-599.

[20] B. Stojkoska, D. Solev, and D. Davcev, "Data prediction in WSN using variable step size LMS algorithm," in Proceedings of the 5th International Conference on Sensor Technologies and Applications, 2011.

[21] M. Wu, L. Tan, and N. Xiong, "Data prediction, compression, and recovery in clustered wireless sensor networks for environmental monitoring applications," Information Sciences, vol. 329, pp. 800-818, 2016.

[22] G. M. Dias, B. Bellalta, and S. Oechsner, "The impact of dual prediction schemes on the reduction of the number of transmissions in sensor networks," Computer Communications, vol. 112, pp. 58-72, 2017.

[23] A. Jain, E. Y. Chang, and Y.-F. Wang, "Adaptive stream resource management using kalman filters," in Proceedings of the 2004 ACM SIGMOD international conference on Management of data. ACM, 2004, pp. 11-22.

[24] G. Wang, H. Wang, J. Cao, and M. Guo, "Energy-efficient dual prediction-based data gathering for environmental monitoring applications," in Wireless Communications and Networking Conference, 2007. WCNC 2007. IEEE. IEEE, 2007, pp. 3513-3518.

[25] M. A. Raja and V. Malathi, "An LMS Based Data Reduction Technique for Energy Conservation in Wireless Sensor Network(WSN)," International Journal of Computer Technology and Applications, vol. 3, no. 4, 2012.

[26] F. A. Aderohunmu, G. Paci, D. Brunelli, J. D. Deng, L. Benini, and M. Purvis, "An application-specific forecasting algorithm for extending wsn lifetime," in Distributed Computing in Sensor Systems (DCOSS), 2013 IEEE International Conference on. IEEE, 2013, pp. 374-381.

[27] F. Liu and Y. Chang, "An energy aware adaptive kernel density estimation approach to unequal clustering in wireless sensor networks," IEEE Access, 2019.

[28] Y. Wu, R. M. Rangayyan, Y. Zhou, and S.-C. Ng, "Filtering electrocardiographic signals using an unbiased and normalized adaptive noise reduction system," Medical Engineering \& Physics, vol. 31, no. 1, pp. $17-26,2009$.

[29] J. Hill, R. Szewczyk, A. Woo, S. Hollar, D. Culler, and K. Pister, "System architecture directions for networked sensors," ACM SIGOPS operating systems review, vol. 34, no. 5, pp. 93-104, 2000.

[30] V. Raghunathan, C. Schurgers, S. Park, and M. B. Srivastava, "Energyaware wireless microsensor networks," IEEE Signal processing magazine, vol. 19, no. 2, pp. 40-50, 2002

[31] M. Li, D. Ganesan, and P. Shenoy, "PRESTO: Feedback-driven data management in sensor networks," IEEE/ACM Transactions on Networking (TON), vol. 17, no. 4, pp. 1256-1269, 2009.

[32] J. N. Al-Karaki and A. E. Kamal, "Routing techniques in wireless sensor networks: a survey," IEEE wireless communications, vol. 11, no. 6, pp. 6-28, 2004.

[33] M. M. Afsar and M.-H. Tayarani-N, "Clustering in sensor networks:
A literature survey," Journal of Network and Computer Applications, vol. 46, pp. 198-226, 2014

[34] L. M. Candanedo, V. Feldheim, and D. Deramaix, "Data driven prediction models of energy use of appliances in a low-energy house," Energy and Buildings, vol. 140, pp. 81-97, 2017.

[35] S. De Vito, E. Massera, M. Piga, L. Martinotto, and G. Di Francia, "On field calibration of an electronic nose for benzene estimation in an urban pollution monitoring scenario," Sensors and Actuators B: Chemical, vol. 129, no. 2, pp. 750-757, 2008.

[36] G. Smaragdakis, I. Matta, and A. Bestavros, "SEP: A Stable Election Protocol for clustered heterogeneous wireless sensor networks," in Second International Workshop on Sensor and Actor Network Protocols and Applications (SANPA 2004), Boston, MA, August 2004.

[37] F. Murena, "Air quality nearby road traffic tunnel portals: BTEX monitoring," Journal of Environmental Sciences, vol. 19, no. 5, pp. 578$583,2007$.

[38] R. Snyder, "Leukemia and benzene," International journal of environmental research and public health, vol. 9, no. 8, pp. 2875-2893, 2012.

[39] L. XingGuo, W. JunFeng, and B. LinLin, "LEACH protocol and its improved algorithm in wireless sensor network," in 2016 international conference on cyber-enabled distributed computing and knowledge discovery (CyberC). IEEE, 2016, pp. 418-422.

[40] M. Tong and M. Tang, "LEACH-B: an improved LEACH protocol for wireless sensor network," in 2010 6th international conference on wireless communications networking and mobile computing (WiCOM). IEEE, 2010, pp. 1-4.

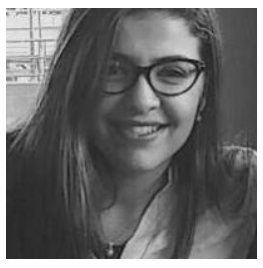

Yasmin Fathy is a Research Associate at the Computer Science Department, University College London (UCL) and a Fellow of the Higher Education Academy. She received her PhD from the Institute of Communication Systems (ICS) at the University of Surrey and MSc in Artificial Intelligence (AI) from AI Lab at Vrije Universiteit Brussel (VUB) in Belgium. Her research interests include machine learning, Internet of Things, and information search and retrieval (e-mail: y.fathy@ucl.ac.uk).

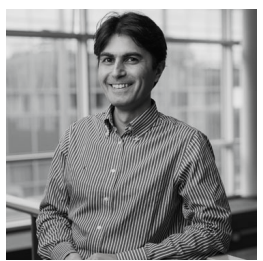

Payam Barnaghi is Professor of Machine Intelligence at the Centre for Vision, Speech and Signal Processing (CVSSP) at the University of Surrey. $\mathrm{He}$ is Deputy Director of the Care Research and Technology Centre at the UK Dementia Research Institute (UK DRI). His research interests include machine learning, healthcare, Internet of Things, and information search and retrieval (email: p.barnaghi@surrey.ac.uk). 


\section{APPENDIX A}

USE-CASE I: EXPERIMENTS ON REAL-WORLD DATASETS

\section{A. Dataset 1}

Fig. 12 ( $a$ and $b$ ) shows the error of both approaches (i.e. with a requested data quality $98 \%$, i.e. $e_{\max }=2$ ). The red circle indicates the sensor readings that have to be transmitted to their $\mathrm{CH}$. The figures indicate that when the prediction error exceeds $\left|e_{\text {max }}\right|$, the sensor node transmits its readings to their $\mathrm{CH}$. As soon as the prediction error decreases at least $w_{s}$ times such that there are enough observations to make a prediction, the sensor node switches to a stand-alone mode. Furthermore, the number of times that a sensor node that runs AM-DR has to transmit data to its $\mathrm{CH}$ (5 times) is lower than the number of times the same sensor node requires when executes the baseline (15 times) between time instances 1540 and 1640 .

Fig. 13 ( $a$ and b) demonstrates the results of AM-DR and baseline approaches. They demonstrate the difference between actual and predicted sensor readings. AM-DR achieves $93 \%$ communication reduction and the baseline achieves $84.5 \%$ while maintaining $98 \%$ data quality; there is a small deviation of 2 degree between the actual and predicted sensor readings. Fig. 13 (a) also depicts how predicted values deviate significantly from actual values in the baseline case that results at increasing the number of transmissions compared to AM-DR (as shown in Fig. 12).

\section{B. Dataset 2}

Fig. 14 (a and b) shows the error of both approaches (i.e. with a requested data quality at most a deviation of $2^{\circ} \mathrm{C}$, i.e. $\left.e_{\max }=2\right)$. The number of times that a sensor node that runs AM-DR has to transmit data to its $\mathrm{CH}$ (28 times) is lower than the number of times the same sensor node requires when executes the baseline ( 65 times) between time instances 1000 and 2000 .

\section{Dataset 3}

When dealing with data drifts where the changes in distribution or statistical properties are unpredictable, an adaptive prediction-based strategy is a crucial step such that filter weights are updated to cope up with the dynamic changes in the sensor observations.

Fig. 15 shows the prediction error of the baseline and AMDR approaches within samples 1200 and 1300. It is clear that there is a significant difference between prediction errors. To this end, outputs of filters and the actual values are plotted in Fig. 16. Comparing Fig. 15 (a) and Fig. 16 (a), it seems that the output of the filter in the baseline approach within samples 1200 and 1300 is near zero (check Fig. 17 (a)). On the other hand, Fig. 16 (b) and Fig. 17 (b) depict the overall filter output (i.e. convex combination of two adaptive filters) comparing to the actual sensor readings. The red circle indicates that a sensor node has to transmit readings to its $\mathrm{CH}$. Overall, this set of experiments show how AM-DR is capable of adapting to the fluctuation of sensor readings and achieves a sufficient trade-off between data quality and data reduction. 


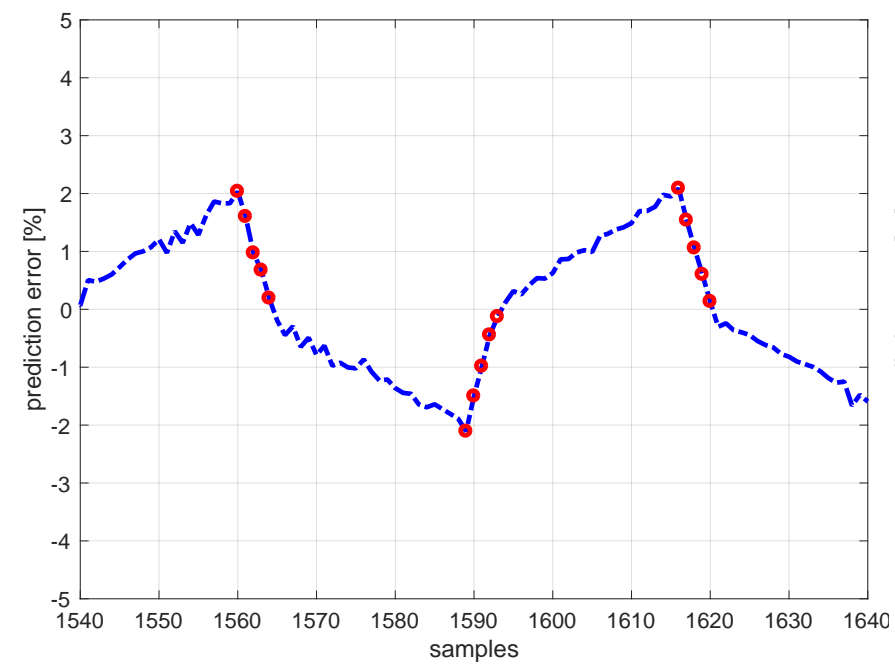

(a) Basline

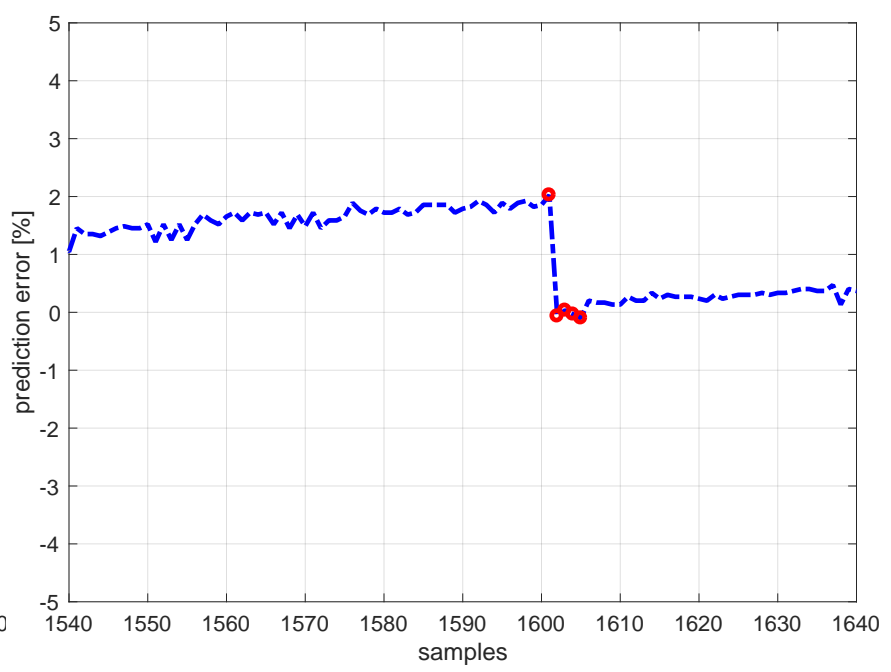

(b) AM-DR

Fig. 12. Dataset 1-Baseline vs AM-DR: prediction error of mote 49 with $e_{\max }=2$

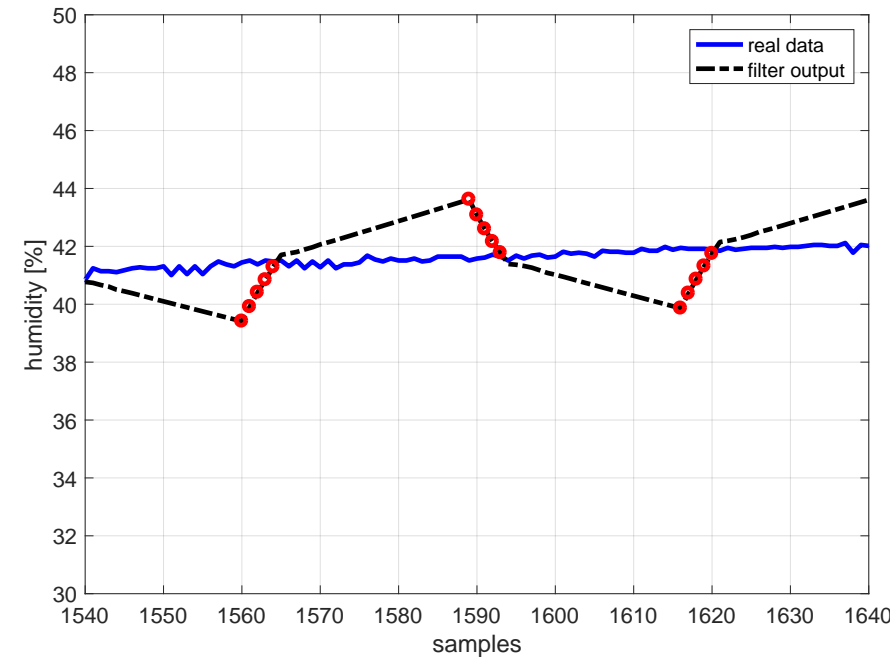

(a) Basline

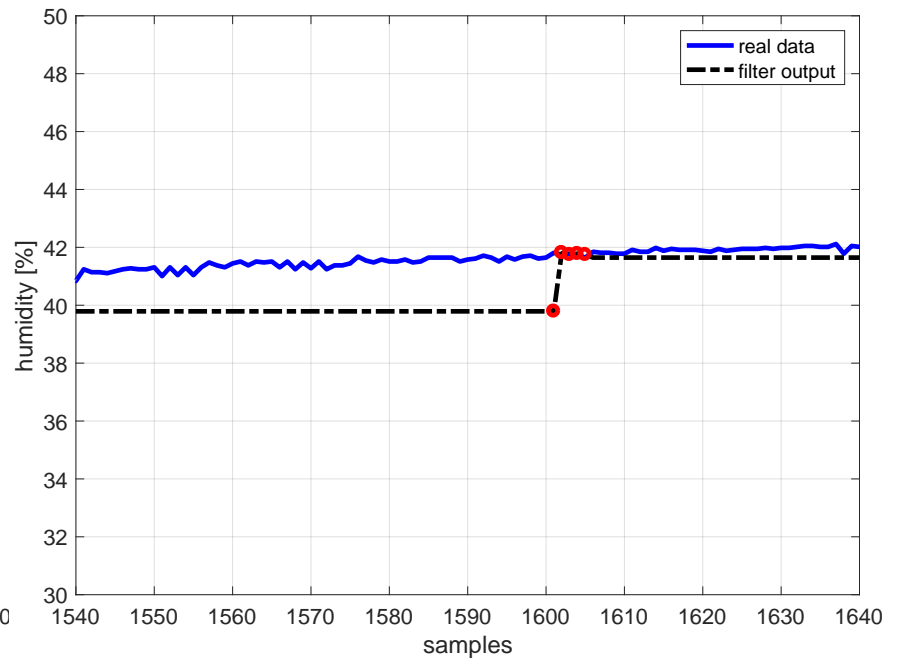

(b) AM-DR

Fig. 13. Dataset 1-Baseline vs AM-DR: real and predicted sensor readings of mote 49 with $e_{\max }=2$

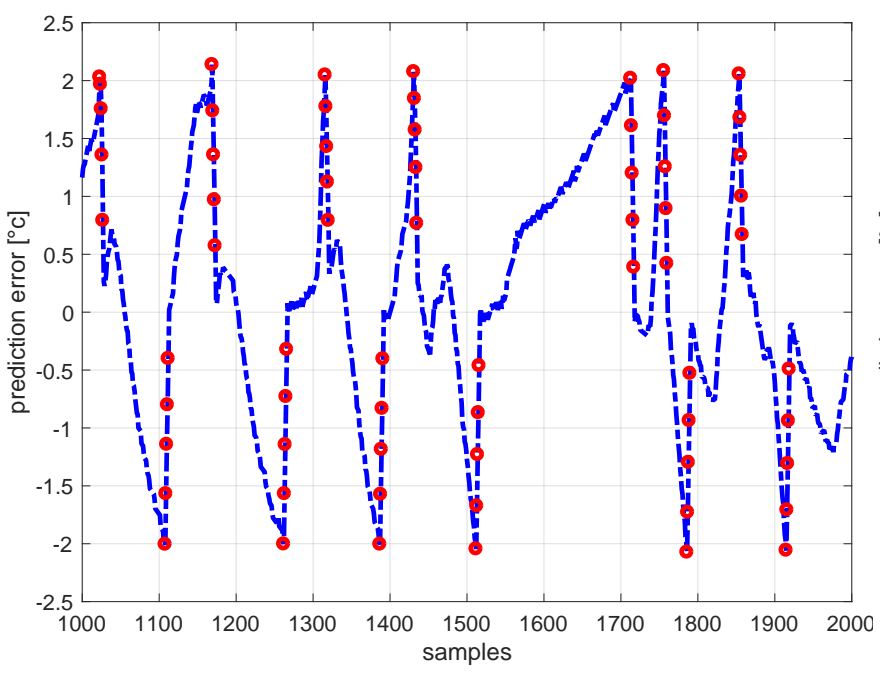

(a) Basline

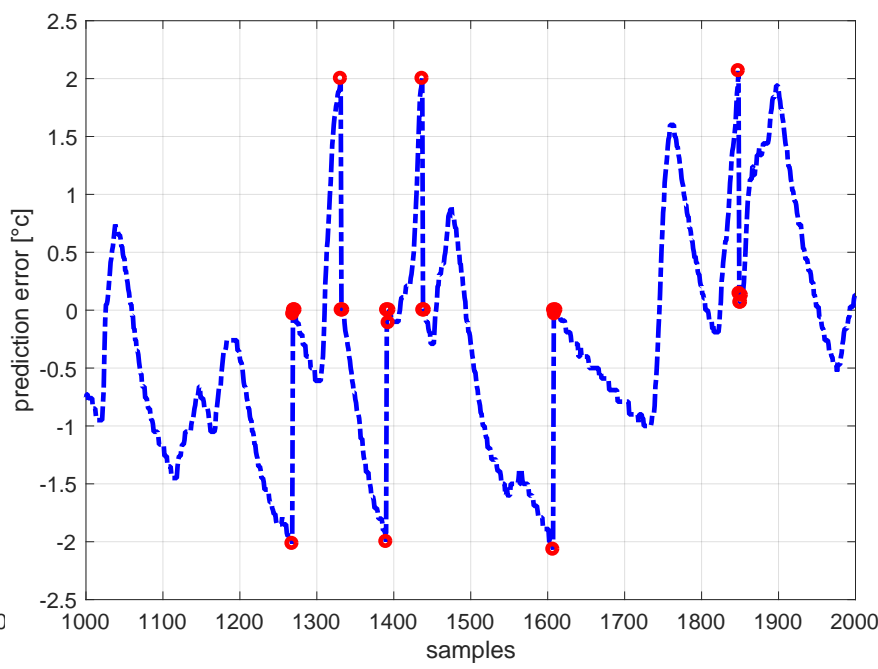

(b) AM-DR

Fig. 14. Dataset 2-Baseline vs AM-DR: prediction error of temperature sensor readings in kitchen with $e_{\max }=2$ 


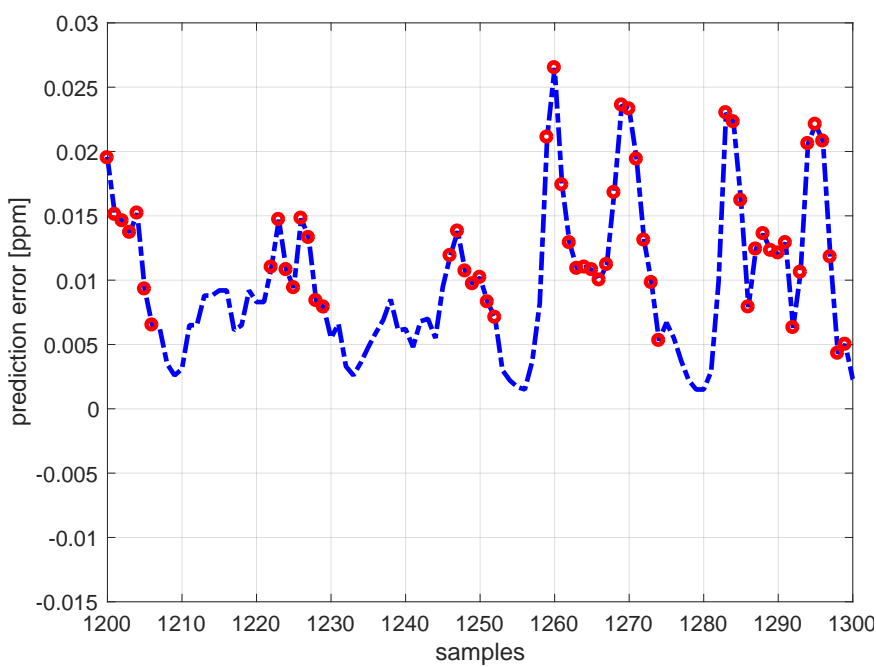

(a) Basline

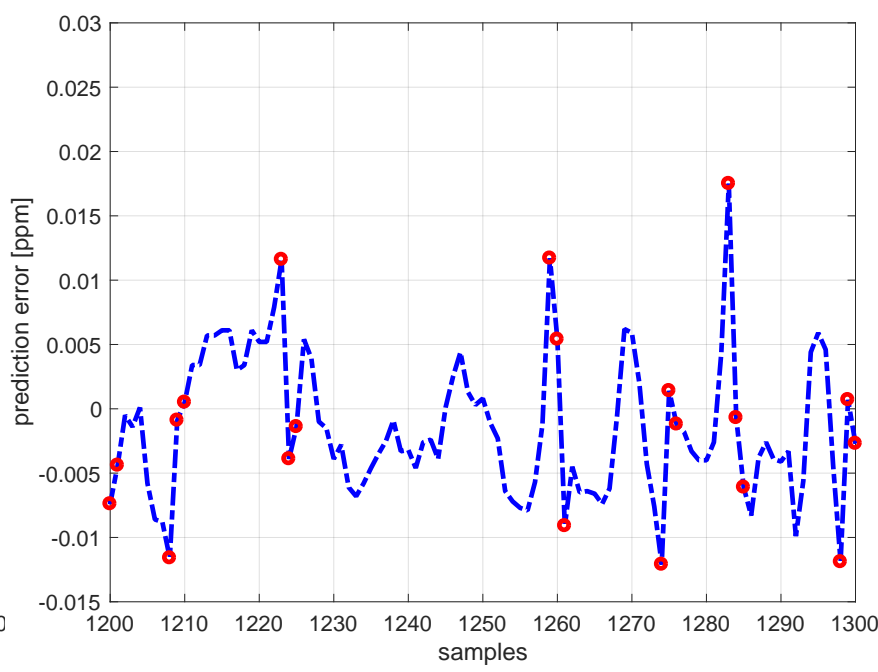

(b) AM-DR

Fig. 15. Dataset 3-Baseline vs AM-DR: prediction error of benzene concentration with $e_{\max }=0.01 \mathrm{ppm}$

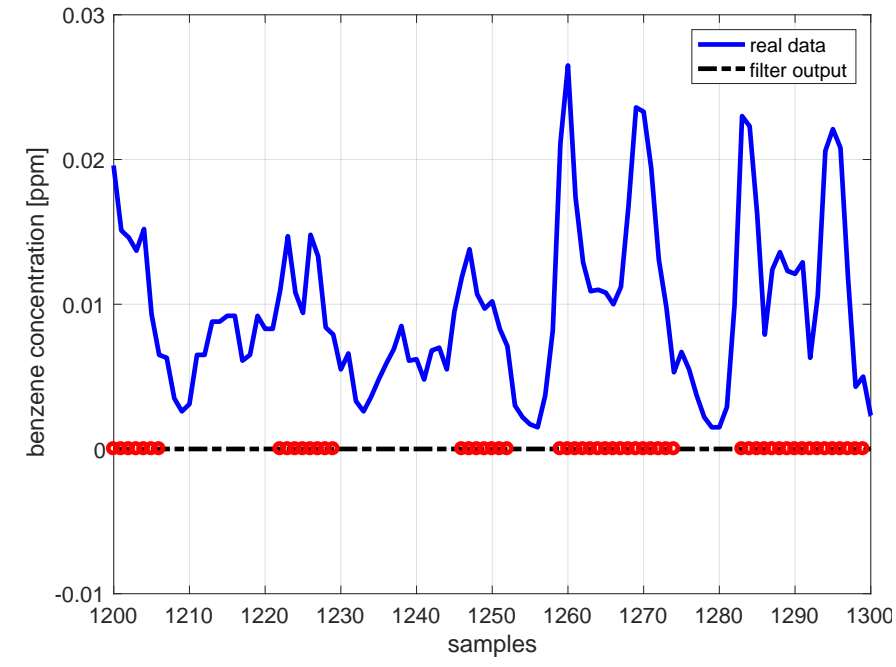

(a) Basline

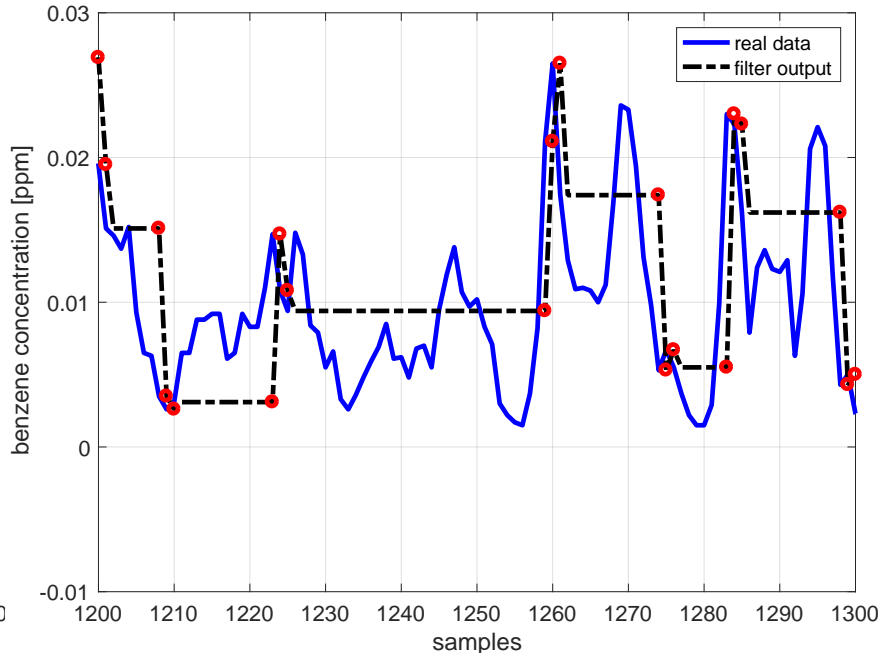

(b) AM-DR

Fig. 16. Dataset 3-Baseline vs AM-DR: real and predicted benzene concentration with $e_{\max }=0.01 \mathrm{ppm}$

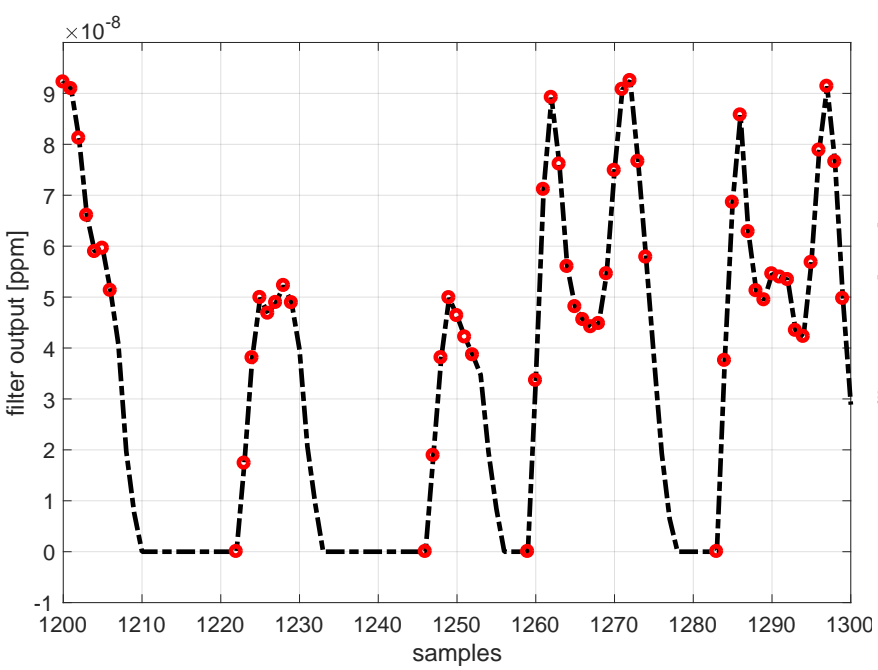

(a) Basline

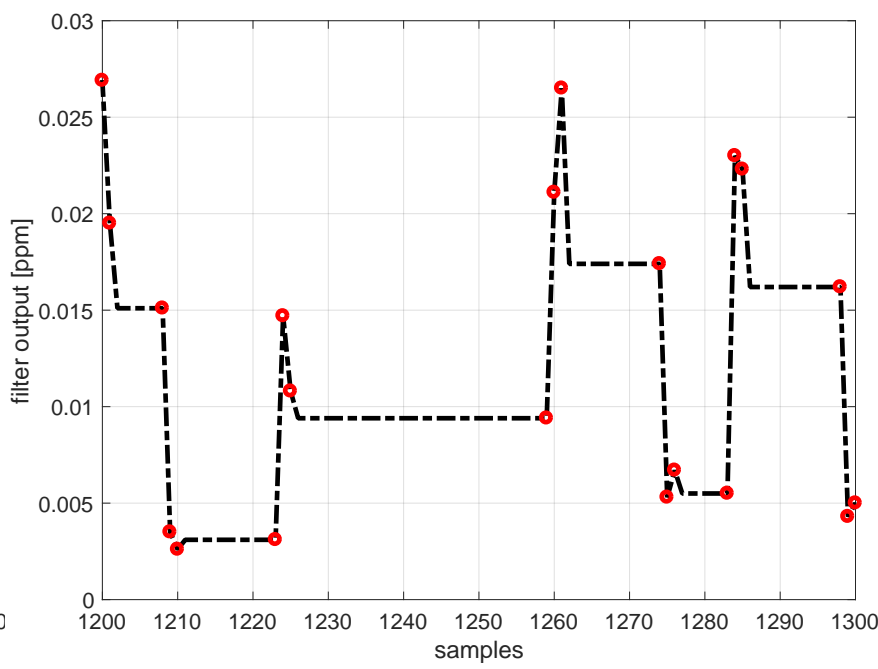

(b) AM-DR

Fig. 17. Dataset 3-Baseline vs AM-DR: filter output of benzene concentration with $e_{\max }=0.01 \mathrm{ppm}$ 